\title{
A Novel Method for Assessing Leg Compartmental Body Composition Using Dual Energy X-ray Absorptiometry
}

\author{
A THESIS \\ SUBMITTED TO THE FACULTY OF THE \\ UNIVERSITY OF MINNESOTA \\ BY
}

Christiana Joy Raymond

\begin{abstract}
IN PARTIAL FULFILLMENT OF THE REQUIREMENTS FOR THE DEGREE OF MASTER OF SCIENCE
\end{abstract}

Advisor: Donald R. Dengel, Ph.D.

May 2016 
(C) Christiana J. Raymond 2016 


\section{Acknowledgements}

It is with great pleasure to have been given the opportunity to prepare this thesis under the advisement of Dr. Donald Dengel, Ph.D. and my committee members, Dr. Tyler Bosch, Ph.D. and Dr. George Biltz, M.D. Thank you, Dr. Dengel, for your mentorship and advice, as well as for providing me with multiple research, writing, and teaching opportunities thus far these past two years. Further, I am very grateful to these three individuals for providing excellent mentorship and feedback throughout the entirety of the writing and statistical analysis processes in producing this thesis. 


\section{Dedication}

To my wonderful family for their continuous support, encouragement, time, and invaluable advice. To my father for demonstrating an incredible work ethic, a love of learning, and a high degree of passion to help people, and to my mother for her unconditional encouragement and positivity. To my siblings for their incredible support and advice, and for serving as inspiring examples of leadership, hard work, and perseverance through challenges faced. Finally, to Zachary Pope for his helpful insights and encouraging words throughout the writing process and for his unconditional support, patience, and positivity. 


\begin{abstract}
PURPOSE: Manual segmentation using dual energy X-ray absorptiometry (DXA) is a valid method for quantifying contralateral tissue asymmetries of the body, but has not been used to quantify body composition in the ipsilateral upper leg compartments. The purpose of this study was to examine the validity and reliability of DXA to quantify fat and lean tissue composition in the anterior and posterior thigh compartments.
\end{abstract} METHODS: Twenty-one (10 male/11 female) college athletes $\left(\bar{X}_{\text {age }}=20.3 \pm 1.3\right.$ yrs $)$ were assessed for total and regional body composition. Segmentation of the anterior and posterior thigh compartments was accomplished by scanning participants lying on their right and left sides using DXA (iDXA, General Electric Medical Systems, Madison, WI, USA). Each scan was manually segmented and analyzed by three technicians using enCore $^{\mathrm{TM}}$ software (platform version 16.0, General Electric Medical Systems, Madison, WI, USA). Custom regions of interest (ROIs) were created from bony landmarks on the femur. In Analysis 1, the proximal border of the custom ROI on the frontal and lateral scans was placed at $40 \%$ of the length from the lateral epicondyle to the greater trochanter. The distal border was placed at $40 \%$ of the length from the lateral epicondyle to the distal end of the lateral malleolus. Lateral and medial borders were placed outside the leg area. To validate the segmentation method, lateral and frontal scan ROI measures of equal leg area for total, fat, and lean masses for each person and each leg were compared. In Analysis 2, proximal and distal ROI borders were placed at $80 \%$ of the length from the lateral epicondyle to the greater trochanter. Lateral and medial ROI borders were placed along the centerline of the shaft of the femur and outside of the leg 
area, respectively, for the anterior ROI compartment and the opposite for the posterior compartment using the custom area drawing tool to quantify compartmental tissue composition. For Analysis 1 we used paired t-tests to assess differences between the measurement methods for each leg (lateral vs. standard position). To assess intra- and inter-rater reliability for Analysis 2, we used intra-class correlation coefficients (ICC) and coefficients of variation (CV) and compared the lateral segmented technique for measures of total, fat, and lean masses. RESULTS: All comparisons of total, fat, and lean masses between the frontal and lateral DXA scans were non-significant (mean differences in right leg: $0.015 \pm 0.12 \mathrm{~kg}, 0.041 \pm 0.19 \mathrm{~kg},-0.0734 \pm 0.22 \mathrm{~kg}$; mean differences in left leg: $0.041 \pm 0.12 \mathrm{~kg},-0.023 \pm 0.17 \mathrm{~kg}, 0.017 \pm 0.23 \mathrm{~kg}$, respectively; $p$ value range: 0.15 to 0.74 ). ICCs were high for all composition measures between- and within-raters, ranging from 0.983 to 0.999 and 0.954 to 0.999 , respectively, with low variation across measures (all CVs: <5\%). CONCLUSION: This study suggests that DXA measures using lateral subject positioning and custom ROIs to assess upper leg total, fat, and lean masses are valid and reliable compared with total-body frontal subject positioning. This novel method allows for anterior/posterior compartmental examination of the aforementioned measures using custom ROIs. Future studies are needed to determine the performance and clinical usefulness of lateral position measures regarding prevention or rehabilitation of injury.

Key Words: Dual-Energy X-ray Absorptiometry, Body Composition, Validity, Reliability 


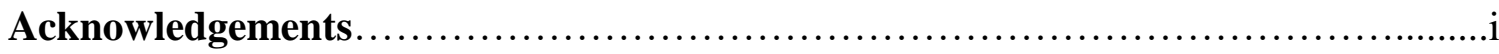

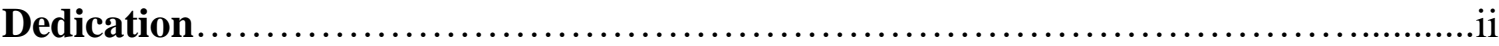

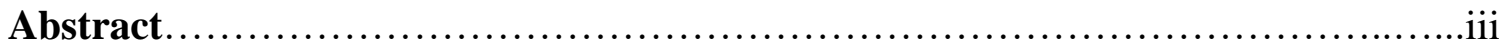

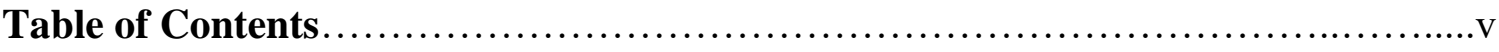

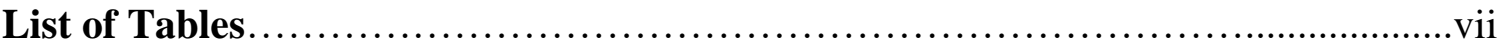

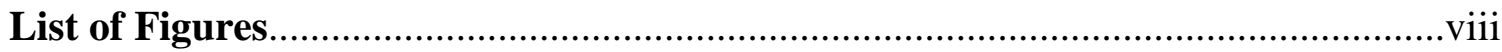

List of Abbreviations.................................................................

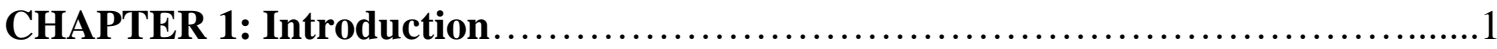

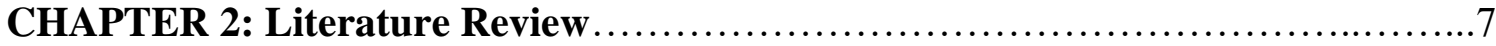

Importance of Body Composition Assessment................................. 8

Simple and Complex Methods of Total and Regional Body Composition..........9

Evolution of DXA Technology.......................................................................16

Comparison of DXA with Other Methods of Body Composition...........................17

Total Body vs. Regional Composition Reliability Studies...........................19

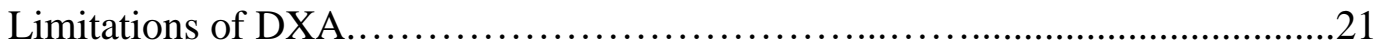

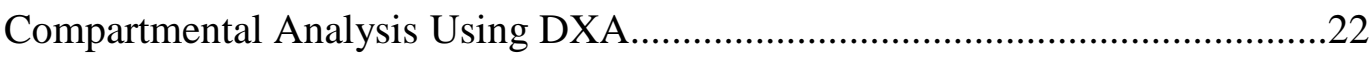

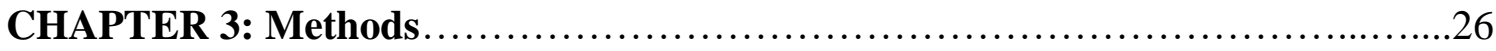

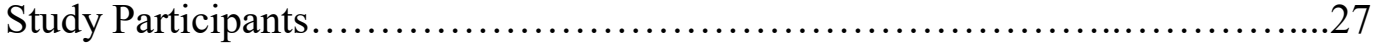

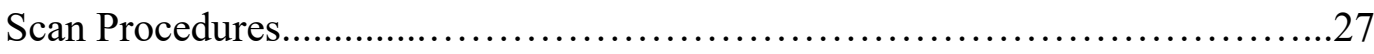

Full Body Composition and Segmentation Quantification......................28

Statistical Analysis..........................................................

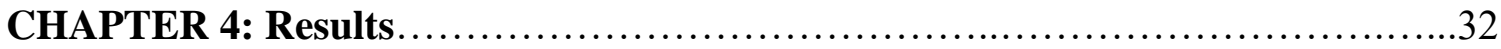

Clinical Characteristics and Body Composition of Study Participants...................33

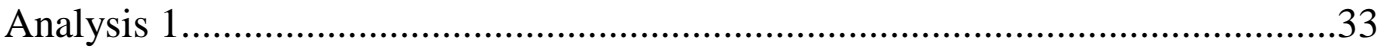

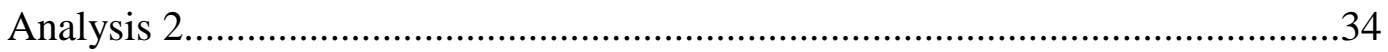

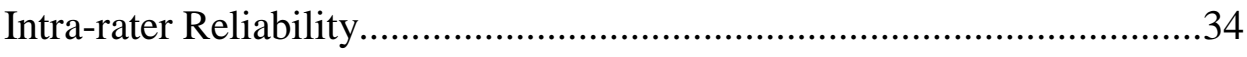

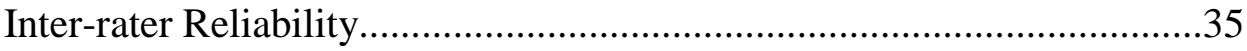

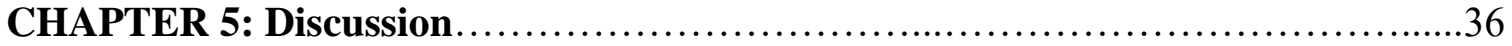




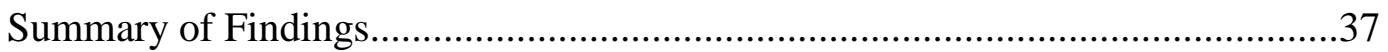

Importance and Implications of Findings.............................................................

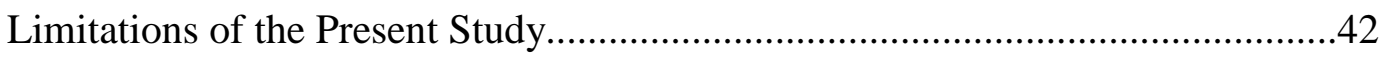

Future Directions...........................................................................................4

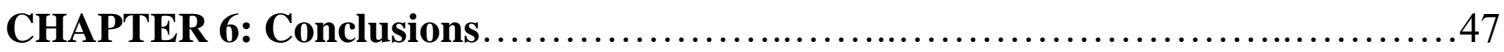

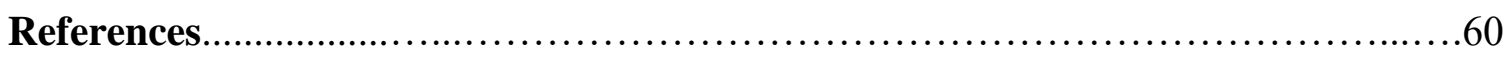




\section{List of Tables}

Table 1. Characteristics of Study Participants........................................................49

Table 2. Total leg measures of bone mineral content (BMC) and total, fat, and lean tissue

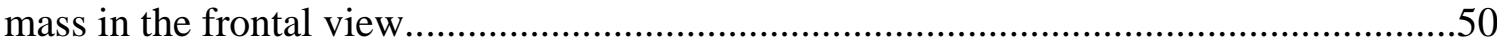

Table 3. Analysis 1: Paired t-test results of the mean difference between total body frontal scan measures and corresponding segmented lateral scan measures

Table 4. Analysis 2: Quantified lateral segmented body measures of total, fat, and lean tissue masses in the total upper leg and anterior/posterior upper leg compartments, averaged over all study participants.

Table 5. Analysis 2: Intra- and inter-rater reliability coefficients of variation for the anterior and posterior compartments of the upper leg and the right and left segmented total upper legs for fat mass, lean tissue mass, and total mass 


\section{List of Figures}

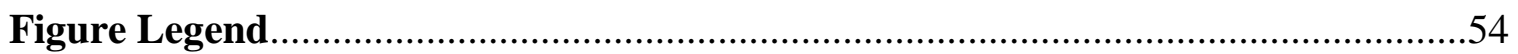

Figure 1. Lateral subject positioning on the DXA scanner.......................................55

Figure 2. DXA scan images displaying total-body frontal subject positioning...............56

Figure 3. DXA scan images displaying lateral subject positioning for the (a) left and (b)

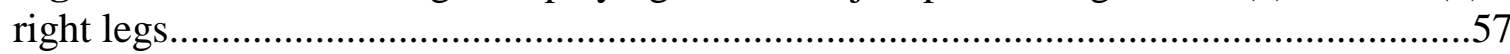

Figure 4. Analysis 1: Comparison of body composition measures between the (a) total body scan in the frontal view and (b) segmented body scan in the lateral view using ROI boxes.

Figure 5. Analysis 2: Left leg DXA scan image with a custom ROI box displaying lateral subject positioning and corresponding body composition measurements for anterior and posterior segmented upper leg compartments. 


\section{List of Abbreviations}

AT: Adipose tissue

SAT: Subcutaneous adipose tissue

VAT: Visceral adipose tissue

BIA: Bioelectrical Impedance Analysis

CT: Computed Tomography

MRI: Magnetic Resonance Imaging

DXA: Dual-energy X-ray absorptiometry

ROI: Region of interest

FOV: Field of view

BMD: Bone mineral density

BMC: Bone mineral content

FM: Fat mass

\%BF: Percent body fat

FFM: Fat-free mass

LM: Lean tissue mass

TBW: Total body water

BMI: Body mass index 


\section{CHAPTER 1: INTRODUCTION}


Body composition assessment has been used in various populations for multiple purposes including, but not limited to: clinically for diagnosis of bone disease (e.g., osteoporosis), skeletal muscle loss (e.g., sarcopenia, cachexia), and for health and nutritional assessments; visceral adiposity evaluation; and in rehabilitation efforts to prevent re-injury (Hart et al., 2015). Typically, body composition estimates are completed with one of three compartment models in mind. The most widely utilized and basic approach to estimate body composition is the two-compartment (2C) model, which divides the body into fat mass (FM) and fat-free mass (FFM) and assumes constant proportions of FFM as protein, water, and minerals (Lee \& Gallagher, 2008). A more complex model, the three-compartment (3C) model, provides bone mineral, fat mass, and lean soft tissue mass measures. Yet, it is the four-compartment (4C) model, which provides total body FM, FFM, bone mineral, and total body water (TBW) measures, that is the most accurate approach to estimate body composition and is the model against which all body composition methods are compared (Lee \& Gallagher, 2008).

Multiple body composition methods utilizing the aforementioned compartment models have been developed including anthropometry, hydrodensitometry, bioelectrical impedance analysis (BIA), computed tomography (CT), magnetic resonance imaging (MRI), and dual-energy X-ray absorptiometry (DXA), among others. DXA, a threecompartment model, is currently considered the gold standard for the quantitative assessment of total and regional body composition and bone mineral density (BMD) in adults and children (Mazess et al., 1990; Haarbo et al., 1991; Lee et al., 2009). Body composition measurement by DXA is also considered the most convenient, safe and non- 
invasive method of measuring bone and soft tissue (i.e., fat and lean muscle) compared with other methods, allowing for its use in a wide range of clinical and performance settings (Pietrobelli et al., 1996; Blake \& Fogelman, 1997). DXA technology is capable of accurately assessing total body and regional body composition (Lee et al., 2009). Current literature reports valid and reliable comparisons of total and regional body composition values in the frontal view against the criterion four-compartment model and other methods of body composition assessment (Pfeiffer et al., 2010; Rothney et al., 2012; Burkhart et al., 2009; Hart et al., 2015).

Currently, full-body DXA scans are used to assess bone mineral content (BMC), FM, and LM. Regions of interest (ROIs) are automatically generated for post-scan analysis using proprietary DXA software, providing segmentation measures of specific regions of the axial (e.g., trunk, abdomen) and appendicular (e.g., upper and lower limbs) skeleton, in addition to visceral adiposity measurements (Heymsfield et al., 1990; Glickman et al., 2004; Kaul et al., 2012; Hart et al., 2015). Recent DXA software developments have allowed for manually generated ROIs to provide quantification of customized sections of the aforementioned regions by manually tracing polygons over the area of interest on a scan (Burkhart et al., 2009). Recent studies (Burkhart et al., 2009; Hart et al, 2015) using DXA have assessed regional quantification of bone mass, FM, and LM in the upper (femur) and lower (shank) segments of the legs in the frontal view, with others assessing contralateral leg symmetry, particularly in athletes (Krzykala \& Leszczynski, 2015). 
To date, no studies have examined measures of bone mass, FM, and LM in the lateral plane using DXA technology. A lateral plane scan would hypothetically allow for comparison of anterior and posterior segments of the same leg. Lateral plane anterior and posterior segments refer to upper leg compartmental tissue composition measures-as seen on a DXA scan - with the assumption that the anterior and posterior ROIs contain a majority of anterior and posterior musculature, respectively, and may contain additional musculature (i.e., adductors). This lateral body composition assessment method would allow for quantification of FM, LM, and total tissue mass in various populations of athletic, diseased, disabled, and elderly individuals. In particular, populations that are limited in standard positioning (e.g. musculoskeletal disorders) may have use for this method for more accurate assessment of change over time.

Lateral segmented body composition measurements may be possible using CT and MRI, as these methods provide high measurement precision due to the capability of these devices to scan specific ROIs and directly assess quality. However, the limitations associated with these methods (i.e., cost, feasibility, and accessibility, in addition to radiation from CT) make their use unlikely for assessing compartment-specific tissue composition. Further, while peripheral quantitative CT (pQCT) allows for quantification of volumetric bone mineral density (as opposed to areal bone mineral density given by DXA), lean mass, and fat mass in specific compartments of the body, it involves high ionizing radiation and is limited in its accuracy compared with MRI and CT (Lee \& Gallagher, 2009; Engelke et al., 2008). 
Therefore, the primary objective of this study was to test a novel method for scanning and segmenting the anterior and posterior compartments of the upper leg from a lateral view using DXA. The specific aims of this project are:

(1) Quantify contralateral and ipsilateral tissue composition;

(2) Validate measures gathered using this novel method with measures obtained using the standard positioning DXA scan;

(3) Demonstrate the inter- and intra-rater reliability of using this novel method to assess compartmental (i.e., anterior/posterior) composition.

The ability to quantify, and longitudinally assess, ipsilateral, compartmental FM and LM would allow for comparisons to be made among athletic, diseased, disabled, and elderly populations and provide information regarding contralateral and ipsilateral imbalances for health, performance, and clinical research.

The following chapters of this thesis include a literature review, methodological explanations, results summary, discussion, and conclusion.

Chapter two provides a comprehensive literature review related to the importance of body composition assessment, methods used to examine body composition, evolution of DXA technology, comparisons of DXA body composition measurements with other body composition measurement methods, and the use of DXA as a validated method in assessing and quantifying fat, lean, and total tissue masses.

Chapter three includes information on the study's methodology including information on the study population, procedures, measurement techniques and equipment, variables of interest, and statistical analyses. 
Chapter four summarizes the results of the study. Comparisons of total tissue mass, fat mass, and lean mass measures between total frontal body and segmented lateral body scans, quantification of total body and compartmental body composition measures, and values demonstrating inter- and intra-rater reliability are included.

Chapter five includes a discussion of study results. Comparisons of body composition measures between the traditional total body and novel segmentation methods of tissue mass quantification, as well as the reliability of measures between- and withinraters, are reviewed. Findings are presented in light of previously published studies related to the current topic.

Chapter six provides final conclusions as well as the clinical and research implications of the study's findings. Specifically, the significance of the novel DXA segmentation method for future improvement and assessment of rehabilitation regimens and prevention of injury are discussed along with recommendations for future studies. 
CHAPTER 2: LITERATURE REVIEW 


\section{Importance of Body Composition Assessment}

The assessment of total-body body composition, including measures of bone mineral density (BMD), bone mineral content (BMC), body fat percentage (\%BF), fat mass (FM), and lean mass (LM), is important not only in the health and the clinical setting, but also in sport performance. It is useful in: the examination of bone density and skeletal mass to monitor osteoporosis and sarcopenia, respectively; strength training programs to prevent injury; and rehabilitation efforts to minimize re-injury risk (Hart et al., 2015). Body composition assessment ideally allows for the detection of tissue imbalances, deficiencies, or excesses of a component thought to be related to health or disease risk (Lee \& Gallagher, 2008). Quantification of LM is of particular interest within the research field to examine sports performance and injury risk, and clinically to evaluate LM changes over time due to disease or age. Various methods of body composition assessment have been developed and utilized to examine total body and regional body composition, including $\mathrm{CT}$, MRI, and DXA, and have been compared with the criterion four-compartment (4C) model of body composition (Lee \& Gallagher, 2008). Currently, however, a lack of literature reports FM and LM quantification of opposing ipsilateral compartments in the assessment of sport- or age-related injury, disease progression, or composition changes due to rehabilitation or weight training programs. It is therefore important to use the most reliable, safe, and cost-effective body composition method available overall_DXA - to examine this relationship for crosssectional and longitudinal analyses, monitoring measurable changes in FM and LM (Lee \& Gallagher, 2008). 


\section{Simple and Complex Methods of Total and Regional Body Composition Assessment}

Multiple body composition methods have been developed—ranging in complexity and ease of use - with many still used currently in the health, performance, and clinical settings. The most widely used methods indirectly measure body composition through prediction equations, and include: anthropometry (i.e., skinfold thickness and circumferences), hydrodensitometry (i.e., underwater weighing), bioelectrical impedance analysis (BIA), computed tomography (CT), magnetic resonance imaging (MRI), and dual-energy X-ray absorptiometry (DXA). In detail, anthropometry, hydrodensitometry, and BIA are based upon the two-compartment (2C) model of body composition-simply providing FM and FFM (i.e., bone and lean soft tissue mass) measures - and are therefore associated with greater errors and less precision than are more complex models of assessment. Therefore, these methods—-particularly anthropometry and BIA—may be used more readily in the field, rather than the clinical, setting. However, CT, MRI, and DXA are based upon the three-compartment (3C) model of body composition-providing bone mineral, FM, and LM measures — and have been validated against the criterion fourcompartment (4C) model (i.e., FM, LM, bone mineral, water), thereby offering greater body composition measurement precision for use in the clinical and laboratory settings.

Two of the earliest methods used to assess body composition were anthropometry and hydrodensitometry (Roubenoff et al., 1991). Anthropometry via skinfold thickness allows for the prediction of $\% \mathrm{BF}$ and provides reliable indices of regional fatness in multiple compartments of the body (Durnin \& Womersley, 1974; Lukaski, 1987). It is also considered one of the best practical methods to measure body composition and can 
provide accurate results for lean subjects, including athletes (Lohman et al., 1997; Wagner \& Heyward, 1999). This method, however, is performed under the following unreliable assumptions: fat-free mass (i.e., FFM; bone and lean muscle) density is constant throughout the body; sites selected for caliper measurements represent the average total-body thickness of subcutaneous adipose tissue (SAT); a relationship between subcutaneous fat and total body fat exists in a constant 1:3 ratio; and SAT in a cross-section of a limb is of constant thickness (Roubenoff et al., 1991; Roche et al., 1996). LM, however, cannot be measured directly using calipers due to the inclusion of intramuscular adipose tissue mass at each measurement site; therefore, LM cannot be predicted reliably using this method (Wells \& Fewtrell, 2006; Roche et al., 1996; Heymsfield et al., 2015). Additionally, anthropometric measures are highly susceptible to inter-observer error, evidenced by this method's high precision error (between 5 and $10 \%)$ and intra-subject variability. Further, anthropometry requires a high degree of investigator expertise to obtain high precision and reliability (Roubenoff et al., 1991). Investigators must be meticulous with site location and measurement of SAT and skin layers. In fact, Hume et al. (2008) found that varying skinfold location by as little as one centimeter gives significantly different values when experienced investigators measure the same participant. Therefore, precise standardized definitions of sites are necessary. Participant age and body fatness may also produce measurement error. Although skinfold measures of body composition may be more reliable in lean individuals, it is more difficult to obtain accurate measures in older populations - with an increased amount of 
loose connective tissue - and obese individuals - a population with larger fat folds (Wagner \& Heyward, 1999).

Hydrodensitometry is used to measure FM, assumes a known density of FM $\left(0.900 \mathrm{~g} / \mathrm{cm}^{3}\right)$ and FFM $\left(1.100 \mathrm{~g} / \mathrm{cm}^{3}\right)$, and was once considered the gold standard for body volume measurements; however, FM and FFM cannot be assessed in isolated regions of the body with this method. Further, it is now known that assumptions of constant FM and FFM density do not always hold true - this is especially evident in children and elderly populations, as proportions of FM as well as protein, mineral, water, and to a lesser extent glycogen (i.e., components of FFM) vary between younger and older individuals, across race, and throughout the duration of fasting, pregnancy, etc. (Heymsfield et al., 2015; Wang et al., 2005). Similar to anthropometry, hydrodensitometry requires a high degree of technician expertise to obtain measures of high precision and reliability (Roubenoff et al., 1991). Hydrodensitometry is fairly precise (approximately 3 to 5\% error) in healthy individuals but prohibits regional body composition assessment (Roubenoff et al., 1991). This method also relies on calculation estimations of bone and skeletal muscle density, as well as residual lung volume, thereby increasing potential measurement error (Heyward \& Gibson, 2014). Finally, measurement error may be introduced given the fact hydrodensitometry requires high participant cooperation in being completely submerged in water while performing a forced maximal exhalation - an unnatural behavior for most individuals. Due to the aforementioned limitations, the use of anthropometry and hydrodensitometry to accurately and reliably assess regional composition for sports performance and 
rehabilitation efforts (i.e., to assess potential LM imbalances between limbs and monitor longitudinal LM changes in response to strength training and rehabilitation programs) is not as useful compared with more complex body composition assessment methods (e.g., CT, MRI, DXA).

BIA depends minimally on investigator skill, is portable, requires very little patient cooperation, and is conveniently used in the performance setting. BIA measures the body's impedance of a small electric current and thus electrical conductivity is greatest in tissues with high fluid and electrolyte content, particularly skeletal muscle (i.e., as opposed to bone or adipose tissue). BIA is therefore limited in its sensitivity to a participant's hydration status, functioning under the assumption that $73 \%$ of the body's FFM is water (Segal et al., 1988; Lukaski, 1989; Esco et al., 2015; Heymsfield et al., 2015). BIA is also subject to error when individuals have participated in recent physical activity because of increased vasodilation-resulting in increased blood flow to skeletal muscle and decreased muscle resistance and impedance - and skin temperature changes, thereby affecting electrical conductivity (Garby et al., 1990). Additionally, female body composition measures can produce greater within-subject variability due to hydration and body weight changes during the menstrual cycle. Gleichauf et al. (1989), however, suggested that the average of multiple measurements taken throughout the menstrual cycle could be used to estimate body composition. Further, although multi-frequency, eight-polar BIA has been shown (Anderson et al., 2012; Esco et al., 2015) to provide reproducible regional assessments of FM and FFM in the arms, legs, and trunk, compared with more complex body composition methods (i.e., DXA), it is not possible to delineate 
regional anatomical boundaries (i.e., manual segmentation). This fact limits BIA's use in clinical, laboratory, and performance settings when assessing diseased, elderly, or athletic populations, among others (Esco et al., 2015). Additionally, the validity of body composition measurements performed by BIA is dependent upon whether the study participant matches the reference population (e.g., obese, elderly, children) from which the prediction equations are obtained (Roubenoff et al., 1991; Buchholz et al., 2004; Fosbøl et al., 2015). Notably, no generalized BIA equations have been developed for athletes using a four-compartment model, resulting in less valid body composition measures in this population using BIA (Moon, 2013). Therefore, despite its small precision error $(<5 \%)$, BIA is not as useful in the assessment of regional compartmental tissue imbalances, nor in the longitudinal tracking of regional tissue mass changes, compared with CT, MRI, or DXA (Roubenoff et al., 1991).

While the aforementioned body composition assessment techniques can be used as simple field methods, more complex methods have been utilized clinically due to greater precision, accuracy, and reliability, and have been validated against the criterion four-compartment model. Notably, the four-compartment model includes measurement, rather than estimation, of total body water (TBW), body mass, body volume, and bone mineral content - particularly allowing for quantification of fat-free mass (i.e., mineral, bone, protein, and water). It is therefore considered the gold standard against which other techniques, such as CT and MRI, can be validated, after cadaver analyses (Lohman et al., 1992; Fuller et al., 1992). When the four-compartment model's use is not feasible (i.e., time constraints using multiple measurement methods), imaging techniques such as CT 
and MRI may be used. These particular techniques offer the ability to assess total and regional body composition to quantify FM and LM in multiple areas. In the research setting, CT and MRI have primarily been used in the quantification of adipose tissue (AT) (i.e., fat) distribution into visceral (VAT), subcutaneous (SAT), or interstitial (i.e., within-muscle) stores (Gallagher et al., 2005), particularly useful when evaluating risk of cardiovascular disease or the metabolic syndrome (Yim et al., 2007). In the 1980s, Sjorstrom $(1986 ; 1991)$ introduced the concept of "total-body multicomponent CT", which allowed for the reliable body composition analysis of multiple body regions. Essentially, imaging "slices" can be taken at each of 22 body sites to examine total-body and regional AT, LM, bone, and other organ and tissue volumes. Similarly, MRI creates "slices" to assess the volume of various tissue types in regional body compartments by detecting hydrogen nuclei, located in water or fat (Wells \& Fewtrell, 2006). MRI also allows for automatic segmentation (more reliable) and manual delineation of different tissues based on signal intensity or morphology (Shen \& Chen, 2008).

Overall, CT and MRI allow for direct visualization of images displaying skeletal muscle and fat within a particular cross-sectional area (Despres et al., 1990). These images can be used to estimate (via mathematical algorithms) the volume of individual muscle groups and total body LM (Heymsfield et al., 1996). In fact, Mitsiopoulos et al. (1998) validated the use of CT and MRI to measure adipose tissue-free skeletal mass (i.e., LM), SAT, and interstitial AT in phantom models against in vitro cadaver analyses. Additional studies (Mourtzakis et al., 2008; Fouladian et al., 2005) have noted the clinical utility and reliability of CT and MRI to accurately quantify FFM in the appendicular and 
axial regions of the body-useful when longitudinally assessing diseased populations (e.g., cachexia, cancer). Additionally, CT allows for detailed and precise regional estimates of bone mass, LM and AT content, particularly of fat infiltration in muscle groups (i.e., inter-muscular fat), and therefore muscle quality implications (Delmonico et al., 2009). Despite these advantages the use of CT and MRI are limited outside of the clinical setting. In detail, the high cost, low accessibility, and long total scanning procedure times (i.e., ranging 10 minutes to two hours) of CT and MRI limit the use of these methods. Additionally, CT's high level of ionizing radiation - up to four times greater than the average annual background radiation exposure — and MRI's difficulty in distinguishing boundaries between tissue layers, its limited measurement accuracy particularly in lean athletes, and its inability to scan patients with cardiac pacemakers or metal implants further limit the use of these methods (Roubenoff et al., 1991; Ackland et al., 2012; National Cancer Institute, 2013). In addition, CT and MRI's accommodation of obese individuals and larger strength athletes is limited in multiple ways. First, the typical 70-centimeter CT gantry aperture diameter (i.e., entry diameter) and 90-centimeter MRI scanner tunnel may leave little room for a larger individual, in addition to CT and MRI's limited scanning field of view (FOV) and a table weight limit between 325 and 440 pounds for MRI (Modica et al., 2011). Further, individuals may feel claustrophobic in the full-body length MRI scanner tunnel. Additionally, slightly different body composition measures may be produced due to the varying parameters of different CT and MRI scanners across models and manufacturers, thereby necessitating the use of the same scanner for multiple scans as well as proper subject positioning. In fact, proper subject 
positioning may be more difficult with obese or larger athletes but is essential to limiting measurement variability in the post-scan analysis. Therefore, despite CT and MRI's capability of accurately assessing adipose tissue (SAT and VAT), muscle mass, muscle cross sectional area, and muscle quality, the aforementioned limitations of CT and MRI have allowed for an additional complex body composition method to become more widely used in the clinical, health, and performance research fields-dual-energy X-ray absorptiometry.

\section{Evolution of DXA Technology}

Dual-energy X-ray absorptiometry (DXA) is a reliable and widely used body composition method, particularly in clinical and laboratory settings, and it has been validated against previous gold standard methods of assessment. Originally based on the principle of bone densitometry, DXA technology has evolved over several decades. Early techniques used X-rays to examine known amounts of BMD (i.e., the bone mineral content (BMC) mass per unit of a particular area) in an aluminum phantom for validation purposes (Blake \& Fogelman, 1997). These early techniques, however, were inconvenient and lacked accuracy (i.e., strength of agreement between the result of a measurement and the true measured value) and precision (i.e., degree to which repeated measurements of the same variable yield the same value) (Toombs et al., 2012).

DXA, introduced in 1987, is a three-compartment model allowing for the reliable differentiation of a subject's BMC, FM, and LM but only solves for two compartments (i.e., soft tissue and bone tissue, or FM and FFM if bone is not present) within each pixel 
(Ganley \& Powers, 2001; Pietrobelli et al., 1996; Lohman, 1996; Kiebzak et al., 2000). Specifically, the most recent DXA technology uses a narrow fan beam collimator to measure body tissue masses via the transmission of X-rays - rather than gamma rays - at two different high and low frequencies, providing a quicker and safer body composition assessment due to minimized ionizing radiation. Depending on an individual's body size, the amount of radiation exposure from DXA is $1 \%$ to $10 \%$ of the radiation emitted by a chest X-ray or CT scan (0.02 to 1.5 mrem vs. 25 to 270 mrem, respectively)—less than $1 \%$ of average annual total background radiation exposure (Lohman \& Chen, 2005; Lee et al., 2009). Further, DXA's ability to isolate body regions and measure tissue masses in each body region during the post-scan analysis allows for more accurate and precise body composition measures (Pietrobelli et al., 1996; Heymsfield et al., 2014). These qualities therefore allow for DXA's use in the clinical and research settings.

\section{Comparison of DXA with Other Methods of Body Composition}

Dual-energy X-ray absorptiometry is currently used to assess total and regional body composition, particularly FM, LM, and \%BF measures. Not only have the most recent DXA models been validated against previous DXA scanning models (Lee et al., 2009; Bilsborough et al., 2014), DXA has also been validated against other established, complex body composition measurement methods, including CT (Visser et al., 1999) and MRI (Shih et al., 2000), among others (Holmes et al., 2005; Lee et al., 2009). Additional studies have examined the relative agreement of body composition values obtained from DXA versus BIA (Bolanowski \& Nilsson, 2001), anthropometry, and hydrodensitometry 
(Kohrt, 1998). The most widely used model to estimate body composition is the twocompartment model, which divides the body into FM and FFM and assumes constant proportions of FFM as protein, water, and minerals. When used, two-compartment estimates should employ population-specific equations (i.e., age, gender, ethnicity, athletic status) if available (Going, 2006). In contrast, the four-compartment model is the criterion method of estimating body composition. The four-compartment model provides values of total body FM, FFM, bone mineral, and total body water and is the model against which all body composition methods are compared-particularly DXA (Lee \& Gallagher, 2008; Bilsborough et al., 2014).

While methods such as hydrodensitometry and BIA, both two-compartment models, allow for the evaluation of total body FM and FFM, DXA offers the advantage of evaluating these components in addition to reliably quantifying bone mass, FM and LM in multiple distinct body regions (Buehring et al., 2014). Further, while CT and MRI have been widely used to assess total and regional LM, DXA has also demonstrated reliability and validity in these measurements. In fact, Visser and colleagues (1999) were among the first to validate manual segmentation using DXA and further compare total leg and total thigh LM measurements between the fan-beam DXA and multi-slice CT methods, resulting in high positive correlations $\left(R^{2}=0.96\right)$. These correlations were similar to subsequent research findings (Levine et al., 2000). Therefore, these findings, in addition to DXA's small cost, greater accessibility, low ionizing radiation levels, non-invasive nature, and quick scan time, make DXA an attractive body composition assessment method across various populations (Kim et al., 2002; Bilsborough et al., 2014). 


\section{Total Body vs. Regional Body Composition Reliability Studies}

Previous research has demonstrated DXA's reliability for measuring total body composition (Bracco et al., 1996; Fields \& Goran, 2000; Prevrhal et al., 2005), and multiple validation studies (Kim et al., 2002; Wang et al., 1999) have concluded that DXA can accurately predict skeletal LM and whole body composition. Further, DXA's accuracy has been validated in multiple studies involving chemical analyses and complex imaging body composition assessment methods. In fact, DXA measurements of FM and LM have been compared with and validated against the four-component model of body composition, described previously, in a diverse array of populations, including young black and white males and females of varying body size, athletic status, musculoskeletal development, and body fatness (Prior et al., 1997; Pietrobelli et al., 1998; Lee \& Gallagher, 2008). Moreover, prediction models for estimating total body LM using DXA have been validated across multiple populations (Lee \& Gallagher, 2008; Prior et al., 1997; Pietrobelli, 1996; Kim et al., 2002; Chen et al., 2007; Wang et al., 1999). Additional studies (Pineau, Filliard, \& Bocquet, 2009; Santos et al., 2010) have found that DXA provides precise and accurate measures of FM, regional FFM, \%BF, and BMD in athletes and is therefore considered a practical criterion method of high performance value (Bilsborough et al., 2014). Thus, DXA's accuracy in quantifying total FM and LM has allowed DXA technology to become the gold standard in assessing body composition in clinical and research settings.

Following the validation of total body composition, the need for regional body composition analysis to assess asymmetries or to provide a more detailed tissue 
composition analysis within particular body regions has been realized. Regional body composition analysis is useful in the evaluation of aging, diseased or disabled individuals (i.e., cachexia, cancer), obesity intervention, sport performance, and rehabilitation efforts. Burkhart and colleagues (2009) were among the first to examine DXA's reliability when segmenting the upper and lower extremities of the body to assess bone mass, FM, and LM (i.e., regional composition measurements). More recently, Hart and colleagues (2015) assessed between-day inter- and intra-rater reliability of manually produced ROIs while segmenting body regions in the frontal view to examine bone and soft tissue (i.e., fat and lean masses). Particular attention was given to appendicular skeletal LM. These studies demonstrated high reliability with small coefficients of variation in the post-scan analysis of the upper and lower regions of the body.

Hart and colleagues (2015) also established successful DXA subject positioning for the upper and lower extremities in the traditional frontal view with CVs of $1.6 \%$ or smaller for all scans, similar to previous studies (Pfeiffer et al., 2010). Standard subject positioning allows for maximum visibility of regional body composition, resulting in high inter- and intra-rater reliability of DXA scan analysis of segmented LM and FM quantification in the frontal view (Hart et al., 2015). Additional body positions have also been examined. Previous studies (Lohman et al., 2009; Lambrinoudaki et al., 1998) have examined DXA's reliability in providing reproducible values of total body FM and LM measures-comparing supine-supine and supine-prone scans. Significant differences resulted between the supine and prone positions in total body measures of FM and LM; it was concluded that differences in subject positioning affect DXA body composition 
measurements due to slight changes in tissue depth and fat distribution between the two positions (Lambrinoudaki et al., 1998). Notably, these studies suggest that variations in body thickness - seen within athletic populations - and the distribution of bone and soft tissue, may interfere with DXA's accuracy. It is therefore suggested that future studies examine additional methods of subject positioning for soft tissue composition assessment, allowing for a lateral view analysis.

\section{Limitations of DXA}

Despite demonstrated reliability, potential limitations of DXA in body composition assessment have been noted: image resolution may be distorted if movements are made while the subject is in the scanner; level of DXA technician experience may affect subject positioning and the resulting resolution of the image captured; biological variability in body composition, weight, height, and joint geometry, thereby affecting the area of the manually-created ROIs for post-scan analysis; inability to directly discern muscle quality; and the manually produced, straight-lined borders (i.e., medial, lateral, superior, inferior) of a ROI (Burkhart et al., 2009; Heymsfield et al., 2015). With DXA's limited capability to outline a ROI using curved lines, soft tissue may be overestimated due to the overlapping of soft tissue included in another ROI (Burkhart et al., 2009). This overlap may result in larger (i.e., less precise) CVs. The most recent literature demonstrates relatively small CVs indicating manual segmentation of DXA images using custom ROIs within the body to be valid and reliable in the quantification of bone, fat, and lean muscle masses (Hart et al., 2013, 2015). Another limitation of the 
DXA technique is its inconsistent measurements reported across scanners manufactured by different companies, such as GE Lunar and Hologic — the two most widely used DXA scanner types (Lohman \& Chen, 2005). Therefore, when testing subjects on more than one occasion, it is important to use the same model of DXA scanner to ensure greater measurement reliability.

\section{Compartmental Analysis Using DXA}

CT, MRI, and DXA have been widely used to quantify total-body, total limb, and regional body (i.e., thigh, calf) LM and are considered the "gold standard" in these assessments. To date, none of these methods have been used to examine differences in ipsilateral, opposing regional compartments (e.g., anterior/posterior compartments of the same upper leg). Indeed, only contralateral (i.e., right vs. left limbs) tissue symmetry analyses have been performed to date. As positioning methods have evolved and newer scan models have been manufactured, accurate and precise FM and LM quantification has become more attainable. Additionally, previous research (Nelson et al., 1996; Hairi et al., 2010) has examined the relationship between regional LM and strength measures to quantify LM and assess muscle quality (i.e., strength per unit of mass). LM quantification is particularly important among diseased (e.g., cachexia), aging (e.g., sarcopenia), and athletic populations, therefore justifying the advancement of research in this area.

In addition, assessment of ipsilateral compartmental differences in the appendicular skeleton (i.e., upper and lower limbs) may be more beneficial in examining injury risk, cause of injury, and the rehabilitative process. Further, Stewart (2001) notes 
the usefulness of DXA - in the standard total frontal body view-to assess longitudinal changes in body composition, as these changes affect the competitive performance of elite athletes. Therefore, the ability to quantify LM and examine longitudinal LM changes (e.g., baseline/pre-injury, post-injury/surgery, before returning to play, or at increments throughout the course of an athlete's career) in opposing ipsilateral (i.e., anterior vs. posterior) compartments of the body - using the segmented lateral scanning methodmay provide a greater breadth of information regarding body composition changes in elite athletes. Further analyses using lateral subject positioning may also prove useful in the clinical and rehabilitation settings in the assessment of particular diseases (e.g., sarcopenia, cachexia) and disorders (e.g., musculoskeletal).

To date, no model exists for accurate and precise segmental analyses of the anterior and posterior compartments of the lower extremity. Previous studies have only assessed tissue mass in regions of the upper and lower leg (e.g., thigh and shank) in the frontal view. Furthermore, to our knowledge, no studies have assessed subject positioning in the lateral view to analyze anterior/posterior compartments. Anterior and posterior segments in the lateral plane refer to the upper leg compartmental measures of tissue composition with the assumption that the anterior and posterior ROIs contain a majority of anterior and posterior musculature, respectively. However, additional upper leg musculature may be included in both regions (e.g., adductor muscles, gracilis, sartorius). In an effort to fill the void of a lateral scan analysis, our laboratory has developed a novel method utilizing DXA to measure FM and LM quantities in the anterior and posterior compartments of the upper leg by positioning participants on their side with their leg 
elevated. In order to assess reliability with this technique, we examined inter- and intrarater reliability-three independent technicians analyzed each scan twice. Body composition scans were performed on a GE Lunar iDXA model with subsequent scan analysis performed using enCORE software (Version 16.0). Using this software, ROIs of the anterior and posterior compartments of the upper left and right legs were manually produced to assess FM, LM, and total tissue mass.

In summary, examining the accuracy and precision of DXA body composition assessments is important when longitudinally monitoring changes in FM and LM. This method may be useful when implementing conditioning regimens to improve health and athletic performance, rehabilitation efforts to prevent athletic injuries and improve LM in the diseased or aging, and nutritional interventions. The purpose of this study, therefore, was to develop a novel scanning method for segmenting the anterior and posterior compartments of the upper leg from a lateral view. This thesis will provide further insight into DXA's validity and reliability in FM and LM quantification in the anterior and posterior upper leg compartments. Establishing validity and reliability of this novel method would allow future research to examine regional and compartmental LM and FM in various types of athletes and clinical populations. This method could further allow for comparisons between injured and non-injured athletes of the same sport as well as comparisons of clinical populations with healthy age-matched controls. Further insight into regional and compartmental LM and FM in the lateral plane may therefore provide greater insight — when compared with the standard total body frontal scan—into physical characteristics (i.e., FM and LM quantity and distribution) of particular types of athletes, 
injury, musculoskeletal status, the aging process, and consequences (e.g., atrophy) of disease. 


\section{CHAPTER 3: METHODS}




\section{Study Participants}

Twenty-one (10 male/11 female) participants ages 18-23 years (mean age $20.3 \pm 1.3 \mathrm{yrs}$ ) were recruited from the University of Minnesota-Twin Cities campus. Participants were healthy and were required to have a body mass index (BMI) $>18 \mathrm{~kg} / \mathrm{m}^{2}$. All participants were required to wear minimal, light clothing free of metallic material. One participant (female) was removed because she only had complete data for one leg (DXA technician deleted the scan files). The study protocol was approved by the University of Minnesota Institutional Review Board and written informed consent was obtained from all participants.

\section{Scan Procedures}

All testing was performed at the Clinical and Translational Science Institute located within the Delaware Clinical Research Unit on the University of Minnesota campus. Height and weight were measured using an electronic scale and wall-mounted stadiometer. BMI was calculated as the body weight in kilograms divided by the height in meters squared. Study participant characteristics are presented in Table 1. Following height and weight measurements, participants underwent three DXA scans, one total body and two leg scans. The leg scans occurred while the participants were lying on their side with foam pads supporting the ankle and thigh of their scanned leg (Figure 1a-b). The pad used to support the thigh was scanned by DXA to ensure it was not recognized by DXA and influence the analyses. In order to create reliable landmarks, prior to scanning each leg, metallic markers were placed on the lateral epicondyle and greater 
trochanter on each individual to identify regions of interest (ROI) for analyses. Thigh girth was measured at the widest point of the thigh segment.

\section{Full Body Composition and Segmentation Quantification}

Participants were asked to arrive in hemostasis and scans were completed between 8:00 am and 12:00 pm. Total body composition was measured using standard procedures on a GE Lunar iDXA system (iDXA, General Electric Medical Systems, Madison, WI, USA) and scans were analyzed using enCore ${ }^{\mathrm{TM}}$ software (platform version 16.0, General Electric Medical Systems, Madison, WI, USA). Standard body positions and procedures were completed for all scans. Upon scan completion, a two-dimensional image was automatically produced for post-scan analysis. All scans were reviewed for accurate placement of the ROI areas by the same technician. Participants first underwent a full-body frontal scan (Figure 2) for quantification of total mass, bone mineral content, fat mass, and lean muscle mass for each leg (Table 2). Participants were then repositioned for the lateral segmentation scans by lying on their side with their feet at the start of the scanning area. The scanned leg was kept straight and positioned down the centerline of the table while the other leg was bent and moved out of the scanning field (Figures 1a-b, 3a-b). The leg being scanned was elevated using foam pads at the ankle and at the widest portion of the upper leg to keep the leg straight, maintain the shape of the muscle, and make the participant comfortable. Using the full-body scan mode, the lateral segmented scans were completed from the foot to the shoulder (Figure 3a-b). After each scan, 
custom ROI boxes were created to quantify tissue composition between the anterior and posterior compartments of the upper leg (Figure 5).

In the first part of the study (i.e., Analysis 1), to compare the effect of leg position on DXA tissue quantification, we created a custom ROI of the same area between traditional frontal and segmented lateral scans within individuals. To determine the optimal ROIs we tested the feasibility of different proportions of the length of each leg segment (femur, shank), creating the largest ROI while minimizing the overlap of other regions of the body (i.e., contralateral leg) and the ankle foam padding (which has an effect on tissue quantification). The proximal border of the custom ROI on the frontal and lateral scans was placed at $40 \%$ of the distance from the lateral epicondyle to the greater trochanter, and the distal border was placed $40 \%$ of the distance from the lateral epicondyle to the lateral malleolus (Figure 4a-b). These three bony landmarks are easily identifiable on the DXA scan, thereby increasing the reliability of the measurements and controlling for differences in leg length. Additionally, we palpated each landmark prior to the scan and marked each with a marker (metal BB) that would show up on the DXA scan to improve the consistency of the measurements. Lateral and medial borders of the ROIs were placed outside the leg circumference, ensuring the inclusion of the entire leg. In the second part of the study, (i.e., Analysis 2), we compared values of FM, LM, and total tissue mass for the total segmented area of the upper leg in addition to the anterior and posterior upper leg compartments between- and within-raters to assess interand intra-rater reliability, respectively. The second analysis used the same scans of participants included in Analysis 1. In the post-scan analysis, two ROI boxes were 
manually created, each encompassing the respective anterior and posterior upper leg compartments. The borders of the anterior ROI were placed at the lateral epicondyle (distal), $80 \%$ of the length between the lateral epicondyle and greater trochanter (proximal), down the shaft of the femur (medial), and outside of the leg area (lateral). The borders of the posterior ROI were placed similarly at the lateral epicondyle (distal), $80 \%$ of the length between the lateral epicondyle and greater trochanter (proximal), down the shaft of the femur (medial), and outside of the leg area (lateral), thereby mirroring the anterior ROI box. The proximal border was placed at $80 \%$ to ensure there was no tissue overlap with any other portion of the body while participants were lying on their side. These ROIs are presented in Figure 5. We quantified the total, fat, and lean tissue masses within the ROI for each leg (Table 4). To assess reliability with this lateral segmented technique, we examined intra- and inter-rater reliability - three independent technicians analyzed each scan twice - by comparing measures of total, fat, and lean mass withinand between-raters.

\section{Statistical Analyses}

Participant characteristics and baseline measurements were calculated for mean \pm standard deviations, when appropriate counts and percentages were presented for categorical variables (Table 1). To validate this new segmentation method, lateral DXA scan measures were compared with total body frontal DXA scan measures of upper leg compartments for fat, lean and total tissue. Paired t-tests were used in Analysis 1 to compare the composition from the lateral DXA scan to the traditional total body frontal 
DXA scan of each participant (Table 3). Intra-class correlation coefficients (ICC) and coefficients of variation (CV) were calculated in Analysis 2 to assess reliability. Interclass correlations measured intra- and inter-rater reliability for the total body and segmented (i.e., anterior/posterior) measurements (Table 5). Paired t-tests, intra-class correlation coefficients (ICC) and coefficients of variation (CV) were calculated using statistical analysis software (SPSS, Version 23.0.0.0; Chicago, IL). The strength of reliability for ICC coefficients was classified in accordance with Hopkins (2000). Coefficients of variation below 5\% were considered highly reliable (Hopkins, 2000). 
CHAPTER 4: RESULTS 


\section{Clinical Characteristics and Body Composition of Study Participants}

Twenty-one college-aged participants (10 male/11 female; mean age $20.3 \pm 1.3$ yrs) were recruited from the University of Minnesota-Twin Cities campus. Participant characteristics and total leg composition by DXA are shown in Tables 1 and 2, respectively. The average BMI for the sample was $26.96 \pm 3.2 \mathrm{~kg} / \mathrm{m}^{2}$ for males and $20.70 \pm 1.3 \mathrm{~kg} / \mathrm{m}^{2}$ for females (range: $21.6-32.0 \mathrm{~kg} / \mathrm{m}^{2}$ for males, $19.0-23.0 \mathrm{~kg} / \mathrm{m}^{2}$ for females) (Table 1). The average height and weight were $1.77 \pm 0.1 \mathrm{~m}(1.84 \pm 0.09 \mathrm{~m}$ for males, $1.69 \pm 0.04 \mathrm{~m}$ for females) and $74.0 \pm 17.4 \mathrm{~kg}(90.2 \pm 10.3 \mathrm{~kg}$ for males, $58.9 \pm 3.6 \mathrm{~kg}$ for females), respectively (Table 1). Average percent body fat (\%BF) for the sample was $16.4 \pm 4.1 \%$ (13.7 $\pm 3.4 \%$ for males, $19.2 \pm 2.5 \%$ for females) (Table 1$)$. Finally, the average total leg bone mineral content (BMC), total leg mass, total leg fat mass, and total leg lean muscle mass for the sample were as follows, respectively: $1342 \pm 340 \mathrm{~g} / \mathrm{cm}^{2}$ (range: 894 $1764 \mathrm{~g} / \mathrm{cm}^{2}$ ), $26.98 \pm 6.3 \mathrm{~kg}$ (range: $20.2-37.4 \mathrm{~kg}$ ), $4.53 \pm 1.2 \mathrm{~kg}$ (range: $2.45-5.74 \mathrm{~kg}$ ), and $20.39 \pm 7.2 \mathrm{~kg}$ (range: $14.28-29.63 \mathrm{~kg}$ ) (Table 2).

\section{Analysis 1}

Manually created ROI boxes for the frontal vs. lateral DXA scans are shown in Figure 4a-b, respectively. Lean muscle, fat, and total tissue mass comparisons between the frontal and lateral DXA scans were all non-significant $(p>0.05)$. Specifically, right leg comparisons of total, fat, and lean muscle masses between the total frontal and segmented lateral DXA scans yielded the following mean differences, respectively: $0.015 \pm 0.12 \mathrm{~kg}$, 
$0.041 \pm 0.19 \mathrm{~kg},-0.074 \pm 0.22 \mathrm{~kg}$ ( $p$-values of $0.599,0.354$, and 0.151$)$. Left leg comparisons yielded the following mean differences, respectively: $0.041 \pm 0.12 \mathrm{~kg}$, $0.0223 \pm 0.17 \mathrm{~kg}, 0.0174 \pm 0.23 \mathrm{~kg}$ ( $p$-values of $0.148,0.547$, and 0.743 ) (Table 3$)$.

\section{Analysis 2}

Reliability coefficients (ICCs) and coefficients of variation (CVs) for intra- and interrater scan analyses resulted in high reliability of body composition measures within the segmented total right and total left upper legs and within the anterior and posterior compartments of the upper leg (Table 5).

\section{Intra-rater Reliability}

Segmented compartmental analysis resulted in strong intra-rater reliability for left and right leg measures of lean muscle mass ( $\mathrm{ICC}=0.954$ to 0.998 ), fat mass ( $\mathrm{ICC}=0.998$ to 0.999), and total mass ( $\mathrm{ICC}=0.991$ to 0.997 ) in the anterior ( $\mathrm{ICC}=0.954$ to 0.999 ) and posterior ( $\mathrm{ICC}=0.997$ to 0.999 ) compartments. Intra-rater coefficients of variation also demonstrated high reliability and precision for lean (CV: 1.20 to $2.69 \%$ ), fat (CV: 1.35 to $2.31 \%$ ), and total mass (CV: 1.12 to $2.38 \%$ ) in the anterior (CV: 1.35 to $2.69 \%$ ) and posterior (CV: 1.12 to $1.75 \%$ ) compartments. Total segmented leg (average ICC $=0.987$, $\mathrm{CV}: 1.91$ to $3.85 \%$ ) measurements resulted in slightly lower reliability, on average, and larger coefficients of variation (i.e., higher \%CV) than those obtained for compartmental (i.e., anterior/posterior) (average $\mathrm{ICC}=0.993, \mathrm{CV}: 1.12$ to $2.69 \%$ ) measurements across 
fat, lean, and total tissue masses. All intra-rater CVs for segmented total composition were $\leq 3.8 \%$ and for compartmental composition were $\leq 2.7 \%$.

\section{Inter-rater Reliability}

Segmented compartmental analysis resulted in high inter-rater reliability for left and right leg measures of lean muscle mass ( $\mathrm{ICC}=0.986$ to 0.996 ), fat mass ( $\mathrm{ICC}=0.998$ to 0.999 ), and total mass (ICC $=0.991$ to 0.998 ) in the anterior $(\mathrm{ICC}=0.991$ to 0.999 ) and posterior (ICC $=0.986$ to 0.999) compartments. Inter-rater coefficients of variation also demonstrated high reliability and precision for lean muscle (CV: 2.60 to 3.90\%), fat (CV: 1.88 to $2.87 \%$ ), and total mass (CV: 1.74 to $3.55 \%$ ) in the anterior (CV: 2.40 to $3.55 \%$ ) and posterior (CV: 1.74 to $3.12 \%$ ) compartments. Anterior and posterior segmented (ICC $=0.986$ to $0.999 ; \mathrm{CV}: 1.74$ to $3.55 \%$ ) measurements resulted in greater reliability and smaller variation than did total segmented leg (ICC $=0.983$ to 0.993 ; CV: 3.05 to 4.80\%) measurements across fat, lean muscle, and total tissue masses. All inter-rater CVs for segmented total composition were $\leq 4.8 \%$ and for compartmental composition were $\leq 3.5 \%$. 


\section{CHAPTER 5: DISCUSSION}




\section{Summary of Findings}

The objectives of this study were to (1) develop and validate a novel method for segmenting the anterior and posterior compartments of the upper leg from a lateral view using DXA; (2) quantify contralateral and ipsilateral upper leg tissue composition; and (3) demonstrate the inter- and intra-rater reliability of using this novel segmentation method to assess compartmental (i.e., anterior/posterior) composition. DXA has been validated as a reliable technique for quantifying bone mass, FM, and LM in total and regional body composition scan analyses in the frontal view in various populations (Mazess et al., 1990; Haarbo et al., 1991; Lee et al., 2009; Bracco et al., 1996; Fields \& Goran, 2000; Fuller et al., 1992; Burkhart et al., 2009; Hart et al., 2015). Presently, DXA is considered the gold standard for body composition assessment in performance and clinical research settings. To our knowledge, no studies have examined ipsilateral body composition in the lateral view to assess FM and LM asymmetries in the anterior and posterior upper leg compartments. Therefore, we analyzed body composition using this novel lateral segmentation method. The analyses yielded the following findings: (1) validity was demonstrated in the comparison of total frontal and segmented lateral body composition measures, as tissue mass values were similar; and (2) high inter- and intrarater reliability was observed in the quantification of LM, FM, and total tissue mass when assessing compartmental body composition measures in the lateral view.

Analysis 1 compared measures of FM, LM, and total tissue mass between the traditional total-body frontal scanning method and the novel segmented lateral scanning method in the right and left upper legs. No significant differences were found between 
the compared measures in either leg of each subject ( $p$-value range: 0.148 to 0.743 ) after paired t-tests were performed (Table 3). However, the current study was likely underpowered to detect a significant difference due to its small sample size of 21 study participants. With the mean difference and standard deviation values of the current study, a sample size of 70 participants would be needed to adequately power the study. These findings, however, provide initial evidence of validity for the use of this novel segmented lateral DXA scanning method in body composition assessments as compared to the traditional total-body frontal DXA scanning method.

Analysis 2 analyzed lateral compartment-specific body composition of the upper leg. In detail, measures of LM, FM, and total tissue mass were quantified for total-body and anterior/posterior right and left upper leg compartments (Table 4). Intra- and interrater reliability of lateral segmented LM, FM, and total tissue mass measures within the upper leg compartments were also assessed across three independent investigators. Reliability analyses of total segmented (i.e., toes-to-shoulder frontal scan; Figure 2) body composition resulted in coefficients of variation $(\mathrm{CVs})$ of $<3.9 \%$ and $\leq 4.8 \%$ within- and between-raters, respectively, while the CVs for compartmental (i.e., anterior/posterior; Figure 5) body composition were $<2.7 \%$ and $<3.6 \%$ for within- and between-raters, respectively (Table 5). The largest within- and between-rater errors were found for FM measures of the total right and total left segmented upper legs (mean within-rater CV: 4.66\%; mean between-rater CV: 3.68\%) (Table 5). Finally, compartmental body composition achieved greater reliability than total segmented body composition measurements for all mass measures. On average, anterior measures produced slightly 
higher within- and between-rater CVs than posterior measures (mean within-rater CVs: $2.09 \%$ vs. $1.42 \%$, respectively; mean between-rater CVs: $2.90 \%$ vs. $2.59 \%$, respectively). All measures of compartment-specific reliability are novel and considered excellent (Hopkins, 2000).

Findings of the current study are consistent with previous literature. Previously reported findings of lower extremity total and regional body composition in the frontal view using GE Lunar iDXA technology have resulted in CVs less than 5\% (CV range: 0.20 and $4.11 \%$ ) (Burkhart et al., 2009). Specifically, average CVs of less than $1.6 \%$ (Hart et al., 2015) and 2.5\% (Rothney et al., 2012) for regional body composition have been found using standard subject positioning in the frontal view. The current study supports the validity and reliability of the segmented lateral DXA scanning method to quantify body composition measures of FM and LM compared with the traditional total body frontal DXA scanning method (Figure 4a-b). In addition, the current study's findings also indicated no significant differences between body composition measures using total (Figure 2) and segmented (Figure 3a-b) body scanning methods with all CVs less than 5\% (Table 5).

\section{Importance and Implications of Findings}

This study demonstrated the validity and reliability of the lateral segmented DXA scanning methodology. Future studies should identify the performance and clinical implications of this new method. Currently, complex body composition assessment methods, including CT, MRI, and DXA, provide contralateral difference measures. Yet, 
examination of ipsilateral, compartmental differences in the upper and lower limbs may be more beneficial in assessing injury risk, causes of injury, and the rehabilitation process. Regarding performance, using this study's novel segmented lateral scanning method to quantify LM in opposing regional body compartments (i.e., anterior/posterior) may allow for the development of LM ratios between compartments for optimal sports performance and rehabilitation. Further, the ability to assess muscle quality and functionality via strength and force output testing in athletic populations would allow for the construction of more efficient and individualized strength training and rehabilitation programs. The demonstrated reliability of the segmented lateral DXA scanning method would further allow for longitudinal tracking of LM and FM changes (e.g., baseline/preinjury, post-injury, before returning to play, etc.) in opposing ipsilateral body compartments of athletes. Lateral segmented longitudinal analysis of tissue changes is important as these changes affect the competitive performance of elite athletes. In fact, Stanforth et al. (2014) reports that elite athletes' performance can be adversely affected by too high or low $\% \mathrm{BF}$, in addition to LM imbalances between opposing compartments of the same limb. Longitudinal tracking of FM and LM over the course of multiple seasons among elite athletes would provide greater insight into causes of injury (i.e., regional imbalances), the recovery process, nutritional interventions, and effective training programs (Stewart, 2001; Bilsborough et al., 2014).

Clinically, this segmented lateral scanning method could be used to assess body composition in elderly, diseased, and disabled populations affected by muscle wasting (e.g., cachexia, sarcopenia) or bone deterioration (e.g., osteoporosis, osteopenia). In 
detail, longitudinal analysis using this novel method would allow for the detection of LM imbalances between upper leg compartments and measurable changes in LM, FM, and BMC/BMD. This method could also be used to monitor improvements in muscle and bone masses over time in response to individualized therapeutic interventions (i.e., pretherapy, midpoint, post-therapy). In this way, loss of LM and strength could be prevented and muscle quality could be improved. Finally, this novel method would allow for muscle quality and functionality assessment in these populations when correlating anterior and posterior compartmental composition measures with measures of strength and force output. Therefore, the ability of this novel segmented lateral scanning method to evaluate opposing compartments of the body ipsilaterally and contralaterally would provide greater insight into the aging and disease processes affecting LM, strength, and quality.

To our knowledge, no reliable technique exists for segmental analyses of the anterior and posterior compartments of the upper leg. In addition, no studies have assessed lateral subject positioning using DXA to analyze compartmental composition. Current literature only reports contralateral comparisons of the upper and lower leg (e.g., thigh and calf muscles) in the frontal view using supine and prone subject positioning (Lambrinoudaki et al., 1998; Hart et al., 2015; Burkhart et al., 2009). The main findings from the current study provide (1) validity support for the use of this segmented lateral DXA scanning method against the total-body frontal scanning method and (2) reliability evidence for the quantification of lateral compartment-specific LM, FM, and total tissue mass using DXA. These findings are important, providing support as an additional 
method for evaluating body composition of various populations for performance research-as in the longitudinal analysis of strength programs for injury risk prevention — and clinical purposes — as in health and disease assessment.

\section{Limitations of the Present Study}

Limitations of the current pilot study include its small sample size, systematic measurement errors in the manual generation of ROIs, and the limited size of the manually generated ROI boxes due to the ankle foam pad. Notably, the small sample size may have contributed to a higher variation in the standard deviation of the mean differences between the total frontal and segmented lateral scans in Analysis 1, as well as a higher variation within- and between- raters reflected in higher CV values in Analysis 2. A larger sample size would have likely decreased the standard deviation of the mean differences and decreased $\mathrm{CV}$ values (i.e., greater precision), thereby providing greater reliability. Yet, despite this small sample size, this study provided evidence to suggest that this method is valid and reliable. Second, although specific instructions detailed the manual production of ROI borders in both analyses, potential slight differences in ROI box measurements may have occurred between the standard total body frontal and segmented lateral scans (i.e., Analysis 1) and for quantification of tissue composition in the anterior and posterior compartments between- and within-raters (i.e., Analysis 2). Thirdly, the foam pad placed at the ankle limited the manual production of the ROI borders in the first analysis. In essence, to avoid the inclusion of the foam pad, the ROI borders were placed at an equidistant superior and inferior to the lateral epicondyle at a 
length that would prohibit inclusion of the ankle foam pad. Further, the post-scan analysis of this novel segmented lateral scanning technique may not be capable of fully separating muscle compartments, thereby including additional muscles in either the anterior or posterior regions (e.g., adductor muscles, etc.)—a limitation of the DXA scanner device. Despite these limitations, no significant differences in body composition measurements were observed between the total-body frontal and segmented lateral scanning methods, and ICC and CV values were highly reliable within- and between-raters. A larger sample size and greater precision of custom ROI borders may demonstrate higher reliability.

\section{Future Directions}

The established validity and reliability of this novel segmented lateral DXA scanning method could prove to be useful in the health, performance, and clinical settings. In the performance setting, current literature notes the utilization of DXA as a practical method to assess an athlete's body composition in the total body frontal plane as it relates to performance and nutritional intervention (Ackland et al., 2012). Nana and colleagues (2014) state multiple reasons as to why DXA is useful in examining standard frontal plane total and regional body composition in various athletic populations. These reasons include: describing physical characteristics across sports or within subgroups of athletes involved in the same sport (e.g., soccer, rugby) and examining an athlete's suitability for a weight class in a weight division sport (e.g., wrestling) (Wittich et al., 2001; Sutton et al., 2009; Clark et al., 2007). Additionally, DXA has been used to examine contralateral leg asymmetries - in the standard total body frontal plane-for 
measures of bone mineral density (BMD), FM, and LM in field hockey players (Krzykala \& Leszczynski, 2015). Therefore, a lateral scan analysis would not only provide information pertaining to athletes' physical characteristics and contralateral symmetry but also ipsilateral symmetry in opposing leg compartments (i.e., anterior vs. posterior). Finally, the assessment of ipsilateral compartmental composition may allow for the development of individualized strength training and rehabilitation programs, further facilitating potentially enhanced sports performance while minimizing re-injury risk (Stewart, 2001; Bilsborough et al., 2014).

Clinically, precise and accurate body composition measurement is important in assessing certain medical conditions (i.e., sarcopenia, cachexia), the aging process, and evaluating interventions (Hairi et al., 2010). Further, segmented DXA body composition assessment methods may be useful in populations where standard DXA positioning remains a significant challenge (e.g. musculoskeletal disorders). Therefore, the ability to quantify LM and FM in specific body compartments using this novel segmented lateral DXA scanning method may allow health professionals to gain a greater understanding of disease characteristics. Additionally, rehabilitation therapists may be able to create more effective therapy programs for diseased and disabled individuals—or elderly individuals recovering from falls or surgery (e.g., joint replacement)— to slow or prevent loss of LM and strength. In addition to the implications of using this segmented lateral scanning method within the field of sport performance, the preceding evidence makes clear that this novel method also has the potential to greatly impact clinical populations. 
Future research should therefore standardize lateral subject positioning and use this method to assess compartmental FM and LM in various types of athletes (i.e., within the same sport and across different sports) and clinical populations. Examinations of this type would allow for comparisons between injured and non-injured athletes of the same sport, in addition to comparisons of clinical populations with healthy age-matched controls. Further insight into regional and compartmental LM and FM in the lateral plane may therefore provide a more in-depth analysis regarding injury, musculoskeletal status, the aging process, and consequences of disease when compared with the standard total frontal body method. Additionally, this novel method may aid in the development of optimal LM ratios between anterior and posterior limb compartments in various athletic and clinical populations. Finally, subsequent comparison of compartmental LM ratios with measures of isokinetic strength and force output may provide additional information regarding ipsilateral compartmental symmetry in terms of muscle mass, quality, and function.

In summary, the use of DXA to investigate a novel method of segmenting the anterior and posterior compartments of the upper leg in the lateral view yielded validation for this technique against the traditional total-body frontal scanning method. High ICCs and relatively low CVs $(<5 \%)$ in $\mathrm{LM}, \mathrm{FM}$, and total tissue mass measurements in the lateral view across three investigators were also found in the current study. These findings indicate that manual segmentation of DXA images using custom ROIs to assess upper leg compartments in the lateral view is a valid and reliable method to evaluate body composition for performance and clinical research purposes. Future studies should 
examine reliability measures using a larger sample size and a diverse array of populations across age category, body size, body fatness, athletic status, and musculoskeletal development. Additionally, future research should establish standardized lateral subject positioning for maximal visibility of anterior and posterior upper leg compartments while minimizing overlap of the contralateral leg. Standardized lateral subject positioning may allow for greater reliability of compartment-specific body composition measures in the quantification of soft tissue and total masses. The novel lateral segmentation method using DXA demonstrates high inter- and intra-rater reliability in the quantification of lower extremity lateral compartmental body composition. This method can therefore be used (1) in cross-sectional analyses and (2) in longitudinal analyses to track changes in FM and LM-useful when implementing training regimens to improve athletic performance, individualizing rehabilitation programs to prevent re-injury and improve muscle quality and functionality, and preserving LM in diseased, disabled, or elderly populations. 


\section{CHAPTER 6: CONCLUSIONS}


In conclusion, we have demonstrated support for the validity and reliability of assessing body composition measures of total, fat, and lean muscle masses in the upper leg using the novel segmented lateral DXA scanning method in comparison with the traditional total-body frontal scanning method. This novel method allows for the assessment of the aforementioned measures in the anterior and posterior upper leg compartments (e.g., anterior and posterior muscles, respectively). Quantification, as well as ipsilateral and contralateral comparisons, of tissue measures in these compartments may allow for optimal tissue mass ratios to be established in the evaluation of sports performance, injury prevention, and rehabilitation efforts. Further, lean tissue mass and fat mass quantification in specific body compartments would allow rehabilitation therapists to individualize therapy programs for diseased and disabled individuals—or elderly individuals recovering from falls or surgery (e.g., joint replacement) - to slow or prevent loss of LM and strength and improve muscle quality. Future clinical and research studies should consider the following: standardizing lateral subject positioning; establishing optimal FM and LM compartmental ratios for sports performance; examining FM and LM ratios in elderly, diseased, and disabled populations for the purpose of designing therapeutic interventions; and comparing tissue measures with measures of strength and force output to assess muscle quality and functionality. These future directions could allow for the ability to predict and prevent sports- and age-related injuries (e.g., hamstring strains, anterior cruciate ligament tears, bone fracture due to falls, etc.) and could therefore improve the health and performance status of individuals. 
Table 1. Characteristics of Study Participants

\begin{tabular}{lccc}
\hline & Male & Female & Total \\
\hline $\mathbf{n}$ & 10 & 11 & 21 \\
Age, $\mathbf{y r}$ & $20.4 \pm 1.4$ & $20.2 \pm 1.3$ & $20.3 \pm 1.3$ \\
Body weight, $\mathbf{~ k g}$ & $90.2 \pm 10.3$ & $58.9 \pm 3.6$ & $74.0 \pm 17.4$ \\
Height, m & $1.84 \pm 0.09$ & $1.69 \pm 0.04$ & $1.77 \pm 0.1$ \\
BMI (kg/m ${ }^{2}$ ) & $27.0 \pm 3.2$ & $20.7 \pm 1.3$ & $23.7 \pm 3.9$ \\
Percent Body Fat (\%) & $13.7 \pm 3.4$ & $19.2 \pm 2.5$ & $16.4 \pm 4.1$ \\
Total Fat Mass (g) & $12206 \pm 4144$ & $10966 \pm 2000$ & $11586 \pm 3230$ \\
Total Lean Mass (g) & $75514 \pm 6526$ & $45965 \pm 2112$ & $60739 \pm 15877$ \\
\hline
\end{tabular}

Abbreviations: BMI, body mass index

Body weight, height, BMI, \% body fat, total fat mass, and total lean mass are presented as mean $\pm \mathrm{SD}$.

${ }^{\wedge} n=21$ (10 males, 11 females) 
Table 2. Total leg measures of bone mineral content (BMC) and total, fat, and lean tissue mass in the frontal view

\begin{tabular}{lccc}
\hline & Male & Female & Total \\
\hline Leg Total BMC $\left(\mathbf{g} / \mathbf{c m}^{2}\right)$ & $1653 \pm 146$ & $1031 \pm 91$ & $1342 \pm 340$ \\
Right Leg BMC $\left(\mathbf{g} / \mathbf{c m}^{2}\right)$ & $825 \pm 84$ & $571 \pm 183$ & $698 \pm 190$ \\
Left Leg BMC $\left(\mathbf{g} / \mathbf{c m}^{2}\right)$ & $828 \pm 68$ & $505 \pm 43$ & $671 \pm 170$ \\
\hline Leg Total Mass $(\mathbf{k g})$ & $32.49 \pm 3.9$ & $21.46 \pm 1.3$ & $26.98 \pm 6.3$ \\
Right Total Mass $(\mathbf{k g})$ & $16.30 \pm 2.1$ & $10.79 \pm 0.7$ & $13.55 \pm 3.2$ \\
Left Total Mass $(\mathbf{k g})$ & $16.2 \pm 1.8$ & $10.69 \pm 0.66$ & $13.44 \pm 3.1$ \\
\hline Leg Fat Mass $(\mathbf{g})$ & $4426 \pm 1617$ & $4641 \pm 705$ & $4533 \pm 1219$ \\
Right Fat Mass $(\mathbf{g})$ & $2226 \pm 857$ & $2321 \pm 340$ & $2273 \pm 636$ \\
Left Fat Mass $(\mathbf{g})$ & $2200 \pm 764$ & $2320 \pm 368$ & $2260 \pm 587$ \\
\hline Leg Lean Mass $(\mathbf{g})$ & $26408 \pm 2681$ & $14364 \pm 4618$ & $20386 \pm 7189$ \\
Right Lean Mass $(\mathbf{g})$ & $13242 \pm 1476$ & $7948 \pm 573$ & $10594 \pm 2906$ \\
Left Lean Mass $(\mathbf{g})$ & $13166 \pm 1227$ & $7843 \pm 573$ & $10595 \pm 2906$ \\
\hline
\end{tabular}

All values reported as means $\pm \mathrm{SD}$. 
Table 3. Analysis 1: Paired t-test results of the mean difference between total body frontal scan measures and corresponding segmented lateral scan measures

\begin{tabular}{ccccc}
\hline Side & Variable & $\begin{array}{c}\text { Mean } \\
\text { Difference } \pm \text { SD }\end{array}$ & $\begin{array}{c}\text { 95\% Confidence } \\
\text { Interval }\end{array}$ & $\boldsymbol{P}$-value \\
\hline Right & Total Mass (kg) & $0.015 \pm 0.12$ & $-0.042,0.071$ & 0.599 \\
& Fat Mass (g) & $40.6 \pm 192$ & $-49.0,130.5$ & 0.354 \\
& Lean Muscle Mass (g) & $-73.5 \pm 220$ & $-176.2,29.3$ & 0.151 \\
\hline \multirow{2}{*}{ Left } & Total Mass (kg) & $0.041 \pm 0.12$ & $-0.016,0.097$ & 0.148 \\
& Fat Mass (g) & $-22.7 \pm 165$ & $-99.9,54.6$ & 0.547 \\
& Lean Muscle Mass (g) & $17.4 \pm 233$ & $-91.9,126.7$ & 0.743 \\
\hline
\end{tabular}

$P$-values and $95 \%$ Confidence Intervals were calculated using paired t-tests of the mean difference \pm SD of each measure. 
Table 4. Analysis 2: Quantified lateral segmented body measures of total, fat, and lean tissue masses in the total upper leg and anterior/posterior upper leg compartments, averaged over all study participants

\begin{tabular}{cccc}
\hline & Male & Female & Total \\
\hline Right Total Mass $(\mathbf{k g})$ & $7.70 \pm 1.4$ & $5.89 \pm 0.5$ & $6.85 \pm 1.4$ \\
Anterior $(\mathrm{kg})$ & $3.06 \pm 0.6$ & $1.9 \pm 0.2$ & $2.52 \pm 0.7$ \\
Posterior $(\mathrm{kg})$ & $4.08 \pm 0.6$ & $2.7 \pm 0.29$ & $3.41 \pm 0.8$ \\
Left Total Mass $(\mathbf{k g})$ & $7.60 \pm 1.4$ & $5.98 \pm 0.5$ & $6.85 \pm 1.3$ \\
Anterior $(\mathrm{kg})$ & $2.78 \pm 0.6$ & $1.89 \pm 0.3$ & $2.35 \pm 0.6$ \\
Posterior $(\mathrm{kg})$ & $3.79 \pm 0.4$ & $2.58 \pm 0.2$ & $3.23 \pm 0.8$ \\
\hline Right Fat Mass $(\mathbf{g})$ & $758 \pm 430$ & $1056 \pm 219$ & $923 \pm 352$ \\
Anterior $(\mathrm{g})$ & $300 \pm 172$ & $331 \pm 124$ & $333 \pm 160$ \\
Posterior $(\mathrm{g})$ & $456 \pm 273$ & $462 \pm 133$ & $482 \pm 225$ \\
Left Fat Mass $(\mathbf{g})$ & $767 \pm 470$ & $1048 \pm 225$ & $924 \pm 372$ \\
Anterior $(\mathrm{g})$ & $257 \pm 157$ & $284 \pm 95$ & $275 \pm 122$ \\
Posterior $(\mathrm{g})$ & $414 \pm 242$ & $440.3 \pm 144$ & $440 \pm 195$ \\
\hline Right Lean Mass $(\mathbf{g})$ & $6696 \pm 1096$ & $4646 \pm 465$ & $5712 \pm 1356$ \\
Anterior $(\mathrm{g})$ & $2672 \pm 492$ & $1568 \pm 228$ & $2117 \pm 666$ \\
Posterior $(\mathrm{g})$ & $3504 \pm 391$ & $2179 \pm 253$ & $2830 \pm 737$ \\
Left Lean Mass $(\mathbf{g})$ & $6574 \pm 1144$ & $4762 \pm 416$ & $5711 \pm 1276$ \\
Anterior $(\mathrm{g})$ & $2423 \pm 555$ & $1545 \pm 304$ & $1997 \pm 626$ \\
Posterior $(\mathrm{g})$ & $3287 \pm 355$ & $2091 \pm 239$ & $2720 \pm 717$ \\
\hline
\end{tabular}

All values reported as mean $\pm \mathrm{SD}$. 
Table 5. Analysis 2: Intra-rater and inter-rater reliability coefficients of variation for the anterior and posterior compartments of the upper leg and the right and left segmented total upper legs for fat mass, lean mass, and total mass.

\begin{tabular}{|c|c|c|c|c|c|c|c|c|c|c|c|c|}
\hline \multirow[b]{2}{*}{ Measure } & \multirow[b]{2}{*}{$\begin{array}{c}\text { Fat } \\
\text { (g) }\end{array}$} & \multirow[b]{2}{*}{$\begin{array}{c}\text { Fat } \\
\text { (g) }\end{array}$} & \multicolumn{3}{|c|}{ Intra-rater Coefficients } & \multirow[b]{2}{*}{$\begin{array}{c}\text { Total } \\
\text { (g) }\end{array}$} & \multirow[b]{2}{*}{$\begin{array}{l}\text { Fat } \\
\text { (g) }\end{array}$} & \multirow[b]{2}{*}{$\begin{array}{c}\text { Fat } \\
(\mathrm{g})\end{array}$} & \multicolumn{3}{|c|}{ Inter-rater Coefficients } & \multirow[b]{2}{*}{$\begin{array}{c}\text { Total } \\
(\mathrm{g})\end{array}$} \\
\hline & & & $\begin{array}{c}\text { Lean } \\
(\mathrm{g})\end{array}$ & $\begin{array}{c}\text { Lean } \\
(\mathrm{g})\end{array}$ & $\begin{array}{c}\text { Total } \\
\text { (g) }\end{array}$ & & & & $\begin{array}{c}\text { Lean } \\
(\mathrm{g})\end{array}$ & $\begin{array}{c}\text { Lean } \\
\text { (g) }\end{array}$ & $\begin{array}{c}\text { Total } \\
\text { (g) }\end{array}$ & \\
\hline Compartment & $\mathbf{C V}$ & ICC & $\mathbf{C V}$ & ICC & $\mathbf{C V}$ & ICC & $\mathbf{C V}$ & ICC & $\mathbf{C V}$ & ICC & $\mathbf{C V}$ & ICC \\
\hline Right Anterior & 1.35 & 0.999 & 2.69 & 0.954 & 1.51 & 0.997 & 2.40 & 0.998 & 2.60 & 0.996 & 2.64 & 0.995 \\
\hline Right Posterior & 1.75 & 0.999 & 1.45 & 0.997 & 1.12 & 0.997 & 1.88 & 0.999 & 3.14 & 0.986 & 1.74 & 0.998 \\
\hline Left Anterior & 2.31 & 0.998 & 2.31 & 0.994 & 2.38 & 0.991 & 2.71 & 0.999 & 3.52 & 0.994 & 3.55 & 0.991 \\
\hline Left Posterior & 1.68 & 0.999 & 1.20 & 0.998 & 1.33 & 0.997 & 2.87 & 0.998 & 2.77 & 0.995 & 3.12 & 0.992 \\
\hline Right Total & 3.50 & 0.992 & 1.91 & 0.989 & 2.22 & 0.984 & 4.51 & 0.993 & 3.19 & 0.988 & 3.05 & 0.986 \\
\hline Left Total & 3.85 & 0.989 & 2.19 & 0.988 & 2.38 & 0.982 & 4.80 & 0.990 & 3.90 & 0.985 & 3.70 & 0.983 \\
\hline
\end{tabular}




\section{Figure Legend}

Figure 1. Lateral subject positioning on the DXA scanner

Figure 2. DXA scan images displaying total-body frontal subject positioning

Figure 3. DXA scan images displaying lateral subject positioning for the (a) left and (b) right legs

Figure 4. Analysis 1: Comparison of body composition measures between the (a) total body scan in the frontal view and (b) segmented body scan in the lateral view using ROI boxes. Proximal and distal ROI borders were placed a percentage distance from the lateral epicondyle to the greater trochanter and lateral malleolus, respectively.

Figure 5. Analysis 2: Left leg DXA scan image with a custom ROI box displaying lateral subject positioning and corresponding body composition measurements for anterior and posterior segmented upper leg compartments 
Figure 1. Lateral subject positioning on the DXA scanner

$\mathrm{a}$

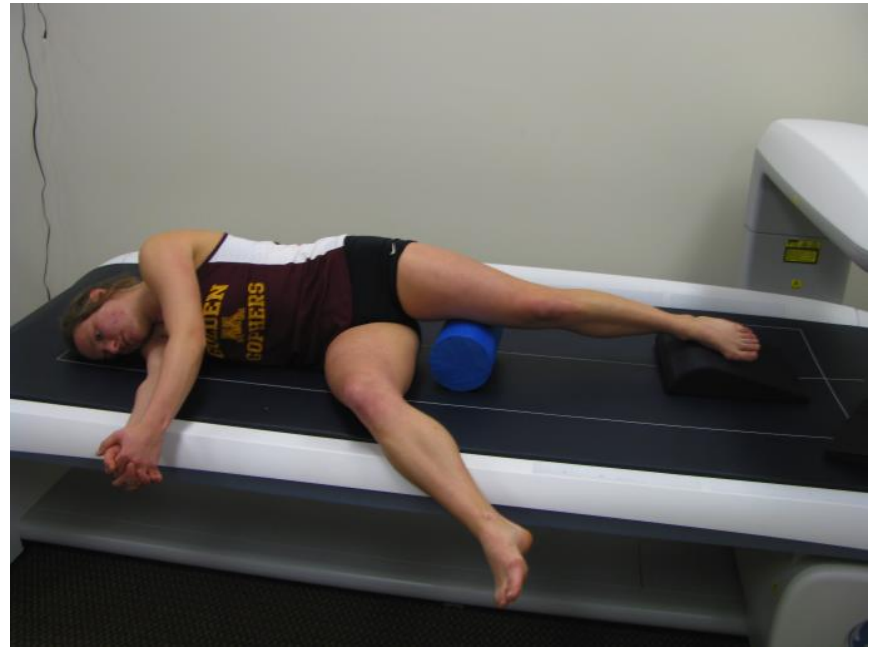

b

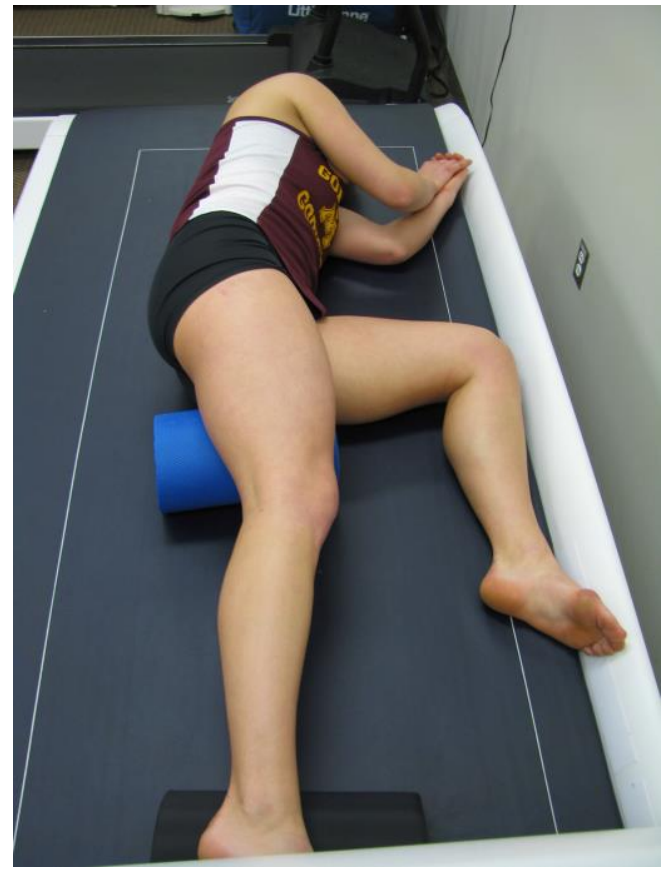


Figure 2. DXA scan images displaying total-body frontal subject positioning
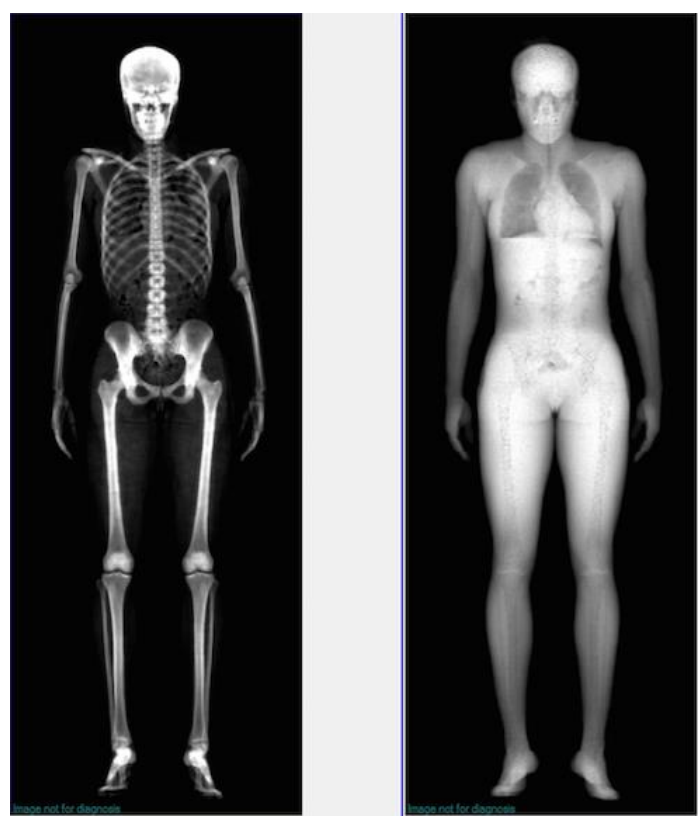
Figure 3. DXA scan images displaying lateral subject positioning for the (a) left and (b) right legs

a

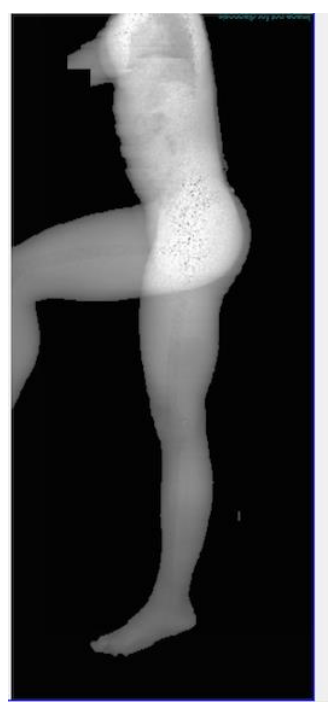

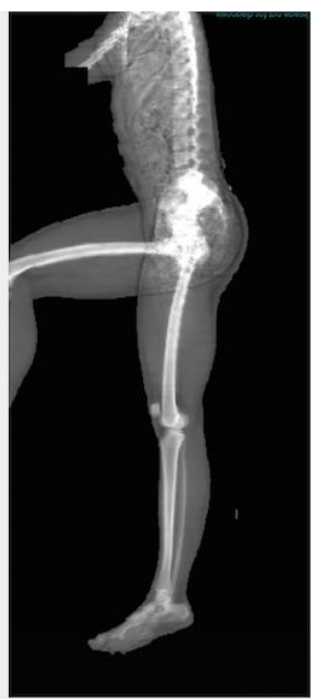

b

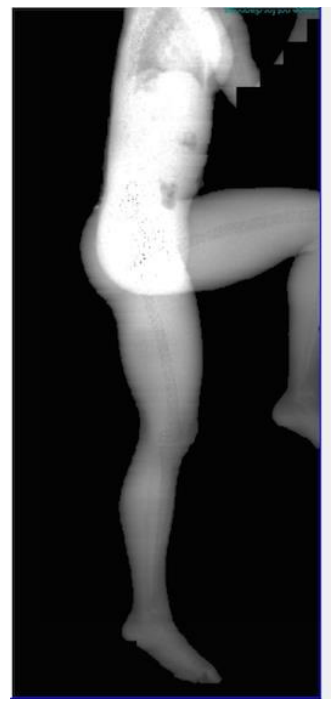

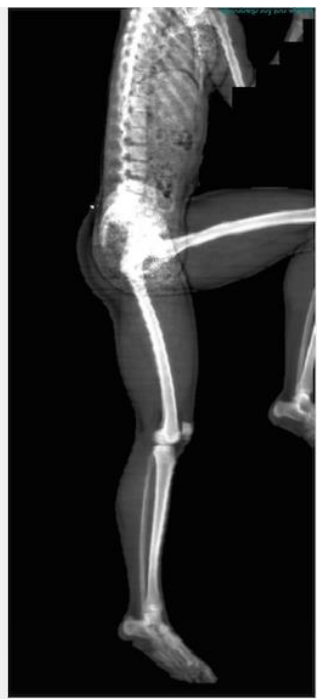


Figure 4. Analysis 1: Comparison of body composition measures between the (a) total body scan in the frontal view and (b) segmented body scan in the lateral view using ROI boxes

a

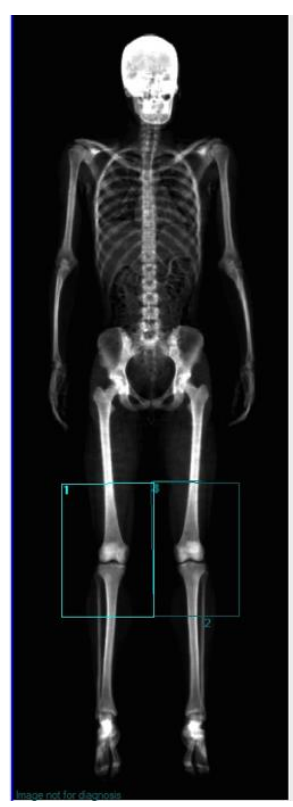

b

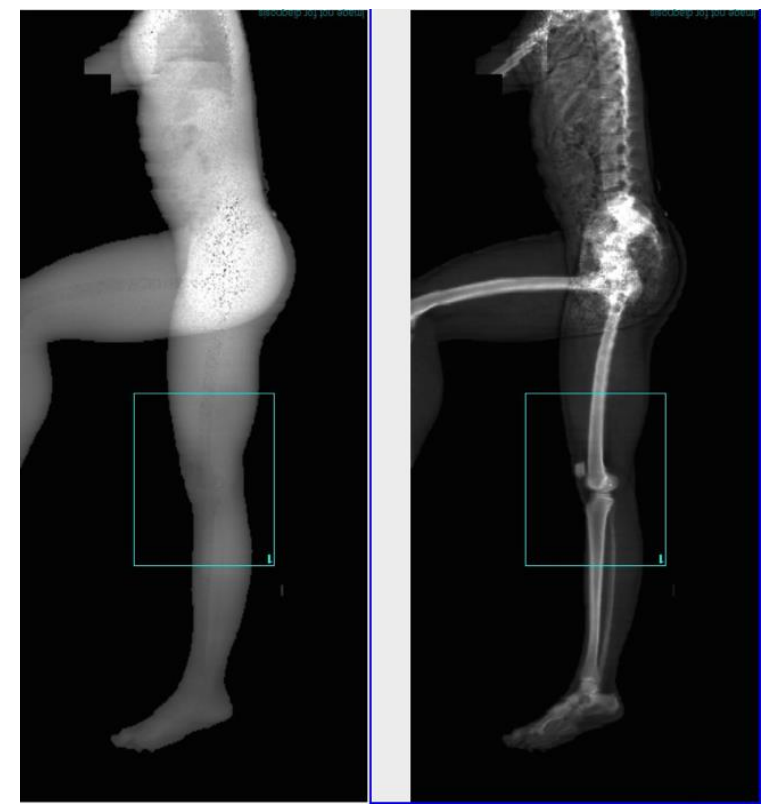

Note: Proximal and distal ROI borders were placed $40 \%$ of the length from the lateral epicondyle to the greater trochanter and lateral malleolus, respectively. 
Figure 5. Analysis 2: Left leg DXA scan image with a custom ROI box displaying lateral subject positioning and corresponding body composition measurements for anterior and posterior segmented upper leg compartments
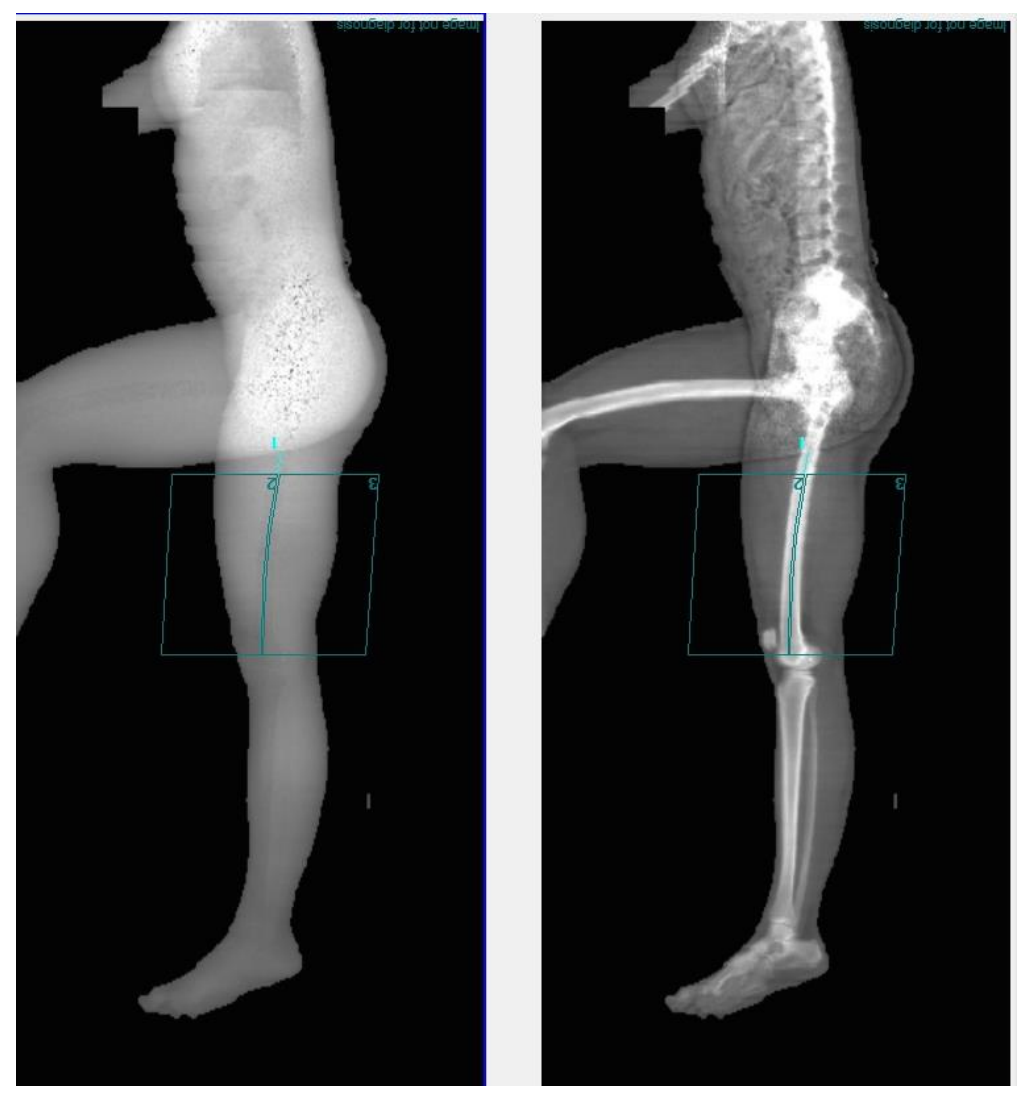


\section{References}

Ackland, T.R., Lohman, T.G., Sundgot-Borgen, J., Maughan, R.J., Meyer, N.L., Stewart, A.D., \& Muller, W. (2012). Current status of body composition assessment in sport. Journal of Sports Medicine, 42(3), 227-249.

Anderson, L.J., Erceg, D.N., \& Schroeder, E.T. (2012) Utility of multifrequency bioelectrical impedance compared with dual-energy $\mathrm{x}$-ray absorptiometry for assessment of total and regional body composition varies between men and women. Nutrition Research Journal 32(7), 479-485.

Bilsborough, J.C., Greenway, K., Opar, D., Livingstone, S., Cordy, J., \& Coutts, A.J. (2014). The accuracy and precision of DXA for assessing body composition in team sport athletes. Journal of Sports Sciences, 32(19), 1821-1828.

Blake, G.M, \& Fogelman, I. (1997). Technical principles of dual energy X-ray absorptiometry. Seminars in Nuclear Medicine, 27(3), 210-228.

Bolanowski, M., \& Nilsson, B.E. (2001). Assessment of human body composition using dual-energy x-ray absorptiometry and bioelectrical impedance analysis. Medical Science Monitor, 7(5), MT1029-1033.

Buchholz, A. C., Bartok, C., \& Schoeller, D. A. (2004). The validity of bioelectrical impedance models in clinical populations. Nutrition in Clinical Practice, 19(5), 433-446.

Buehring, B., Krueger, D., Libber, J., Heiderscheit, B., Sanfilippo, J., Johnson, B., Haller, I., \& Binkley, N. (2014). Dual-energy X-ray absorptiometry measured regional body composition least significant change: Effect of region of interest and gender in athletes. Journal of Clinical Densitometry, 17(1), 121-128.

Burkhart, T.A., Arthurs, K.L., \& Andrews, D.M. (2009). Manual segmentation of DXA scan images results in reliable upper and lower extremity soft and rigid tissue mass estimates. Journal of Biomechanics, 27(3), 197-206.

Bracco, D., Thiebaud, D., Chiolero, R.L., Lamdry, M., Burckhardt, P., \& Schutz, Y. (1996). Segmental body composition by bioelectric impedance and DEXA in humans. Journal of Applied Physiology, 81, 2580-2587.

Chen, Z., Wang, Z., Lohman, T., Heymsfield, S. B., Outwater, E., Nicholas, J. S., Bassford, T., LaCroix, A., Sherrill, D., Punyanitya, M., Wu, G., \& Going, S. (2007). Dual-energy X-ray absorptiometry is a valid tool for assessing skeletal muscle mass in older women. The Journal of nutrition, 137(12), 2775-2780.

Clark, R.R., Sullivan, J.C., Bartok, C.J., \& Carrel, A.L. (2007). DXA provides a valid minimum weight in wrestlers. Medicine \& Science in Sports and Exercise, 39, 2069-2075.

Delmonico, M.J., Harris, T.B., Visser, M., Park, S.W., Conroy, M.B., Velasquez-Mieyer, P., Bourdreau, R., Manini, T.M., Nevitt, M., Newman, A.B., \& Goodpaster, B.H. (2009). Longitudinal study of muscle strength, quality, and adipose tissue infiltration. The American Journal of Clinical Nutrition, 90, 1579-1585.

Despres, J.P., Moorjani, S., Lupien, P.J., Tremblay, A., Nadeau, A., \& Bouchard, C. (1990). Regional distribution of body fat, plasma lipoproteins, and cardiovascular 
disease. Arteriosclerosis, 10, 497-511.

Durnin, J.V., \& Womersley, J. (1974). Body fat assessed from total body density and its estimation from skinfold thickness: measurements on 481 men and women aged from 16 to 72 years. British journal of nutrition, 32(01), 77-97.

Engelke, K., Adams, J.E., Armbrecht G., Augat, P, Bogado, C.E., Bouxsein, M.L., Felsenberg, D., Ito, M., Prevrhal, S., Hans, D.B., \& Lewiecki, E.M. (2008). Clinical use of quantitative computed tomography and peripheral quantitative computed tomography in the management of osteoporosis in adults: the 2007 ISCD Official Positions. Journal of Clinical Densitometry, 11(1), 123-162.

Esco, M.R., Snarr, R.L., Leatherwood, M.D., Chamberlain, N.A., Redding, M.L., Flatt, A.A., Moon, J.R., Williford, H.N. (2015). Comparison of total and segmental body composition using DXA and multifrequency bioimpedance in collegiate female athletes. Journal of Strength and ConditioningResearch, 29(4). 918-925.

Fields, D.A., \& Goran, M.I. (2000). Body composition techniques and the fourcompartment model in children. Journal of Applied Physiology, 89, 613-620.

Fosbøl, M. Ø., \& Zerahn, B. (2015). Contemporary methods of body composition measurement. Clinical physiology and functional imaging, 35(2), 81-97.

Fouladian, M., Korner, U., \& Boasaeus, I. (2005). Body composition and time course changes in regional distribution of fat and lean tissue in unselected cancer patients on palliative care-correlations with food intake, metabolism, exercise capacity, and hormones. Cancer, 103, 189-198.

Fuller, N.J., Jebb, S.A., Laskey, M.A., Coward, W.A, \& Elia M. (1992). Four-component model for the assessment of body composition in humans: comparison with alternative methods, and evaluation of the density and hydration of fat-free mass. Clinical Science, 82, 687-693.

Fuller, N.J., Laskey, M.A., \& Elia, M. (1992). Assessment of the composition of major body regions by dual-energy X-ray absorptiometry (DEXA), with special reference to limb muscle mass. Clinical Physiology, 12, 253-266.

Gallagher, D., Kuznia, P., Heshka, S., Albu, J., Heymsfield, S. B., Goodpaster, B., Visser, M., \& Harris, T. B. (2005). Adipose tissue in muscle: a novel depot similar in size to visceral adipose tissue. The American journal of clinical nutrition, 81(4), 903-910.

Ganley, K.J., \& Powers, C.M. (2001). The use of dual energy X-ray absorptiometry in determining subject-specific anthropometric measures for kinetic analysis during gait. Gait and Posture, 13, 271-272.

Garby, L., Lammert, O., \& Nielsen, E. (1990). Negligible effects of previous moderate physical activity and changes in environmental temperature on whole body electrical impedance. European Journal of Clinical Nutrition, 44, 545-546.

Gleichauf, C.N., \& Roe, D.A. (1989). The menstrual cycle's effect on the reliability of biomedance measurements for assessing body composition. American Journal of Clinical Nutrition, 50, 903-907.

Glickman, S.G., Marn, C.S., Supiano, M.A., \& Dengel, D.R. (2004). Validity and reliability of dual-energy X-ray absorptiometry for the assessment of abdominal 
adiposity. Journal of Applied Physiology, 97, 509-514.

Going, S. (2006). Optimizing techniques for determining body composition. Sports Science Exchange, 19(2), 1-6.

Haarbo, J., Gotfredsen, A., Hassager, C., \& Christiansen, C. (1991). Validation of body composition by dual energy X-ray absorptiometry (DEXA). Clinical Physiology, 11(4), 331-341.

Hairi, N.N., Cumming, R.G., Naganathan, V., Handelsman, D.J., Le Couteur, D.G., Creasey, H., Waite, L.M., Seibel, M.J., \& Sambrook, P.N. (2010). Loss of muscle strength, mass (sarcopenia), and quality (specific force) and its relationship with functional limitation and physical disability: the Concord Health and Ageing in Men Project. Journal of the American Geriatrics Society, 58(11), 2055-2062.

Hart, N.H., Nimphius, S., Spiteri, T., Cochrane, J.L., \& Newton, R.U. (2015). Segmental musculoskeletal examinations using dual-energy X-ray absorptiometry (DXA): Positioning and analysis considerations. Journal of Sports Science and Medicine, 14, 620-626.

Heyward, V.H, \& Gibson, A. (2014). Advanced fitness assessment and exercise prescription (7th ed.). Champaign, IL: Human Kinetics.

Heymsfield, S.B., Smith, R., Aulet, M., Bensen, B., Lichtman, S., Wang, J., \& Pierson, R.N. (1990). Appendicular skeletal muscle mass: Measurement by dual-photon absorptiometry. American Journal of Clinical Nutrition, 52, 214-218.

Heymsfield, S.B., Wang, Z., Baumgartner, R.N., \& Ross, R. (1996). Human body composition: Advances in models and methods. Annual Review in Nutrition, 17, 527-558.

Heymsfield, S.B., Adamek, M., Gonzalez, M.C., Jia, Guang, \& Thomas, D.M. (2014). Assessing skeletal muscle mass: Historical overview and state of the art. Journal of Cachexia Sarcopenia Muscle, 5, 9-18.

Heymsfield, S.B., Gonzalez, M.C., Lu, Jianhua, Jia, G., \& Zheng, J. (2015). Skeletal muscle mass and quality: Evolution of modern measurement concepts in the context of sarcopenia. Proceedings of the Nutrition Society. 1-12. doi:10.1017/S0029665115000129.

Heymsfield, S.B., Ebbeling, C.B., Zheng, J., Pietrobelli, A., Strauss, B.J., Silva, A.M, \& Ludwig, D.S. (2015). Multi-component molecular-level body composition reference methods: Evolving concepts and future directions. Obesity Review, 16(4), 282-294.

Holmes, J.D., Andrews, D.M., Durkin, J.L., \& Dowling, J.J. (2005). Predicting in-vivo, soft tissue masses of the lower extremity using segment anthropometric measures and DXA. Journal of Applied Biomechanics, 21(4), 371-382.

Hopkins, W. (2000). Measures of reliability in sports medicine and science. Sports Medicine, 30(1), 1-15.

Hume, P., Marfell-Jones, M. (2008). The importance of accurate site location for skinfold measurement. Journal of Sports Science, 26, 1333-1340.

Kaul, S., Rothney, M.P., Peters, D.M., Wacker, W.K., Davis, C.E., Shapiro, M.D., \& Ergun, D.L. (2012). Dual-energy X-ray absorptiometry for quantification of 
visceral fat. Obesity, 20(6), 1313-1318.

Kim, J., Wang, Z., Heymsfield, S. B., Baumgartner, R.N., \& Gallagher, D. (2002). Totalbody skeletal muscle mass: Estimation by a new dual-energy X-ray absorptiometry method. American Journal of Clinical Nutrition, 76, 378-383.

Kohrt, W.M. (1998). Preliminary evidence that DEXA provides an accurate assessment of body composition. Journal of Applied Physiology, 84(1), 372-377.

Krzykala, M., \& Leszczynski, P. (2015). Asymmetry in body composition in female hockey players. HOMO - Journal of Comparative Human Biology, 66(4), 379386.

Lambrinoudaki, I., Georgiou, E., Douskas, G., Tsekes, G., Kyriakidis, M., \& Proukakis, C. (1998). Body composition assessment by dual-energy X-ray absorptiometry: Comparison of prone and supine measurements. Metabolism, 47(11), 1379-1382.

Lee, S.Y., \& Gallagher, D. (2008). Assessment methods in human body composition. Current Opinion in Clinical Nutrition and Metabolic Care, 11, 566-572.

Lee, M.K., Koh, M., Fang, A.C., Le, S.N. \& Balasekaran, G. (2009). Estimation of body segment parameters using dual energy absorptiometry and 3-D exterior geometry. $13^{\text {th }}$ International Conference on Biomedical Engineering, 23(5), 1777-1780.

Levine, J.A., Abboud, L., Barry, M., Reed, J.E., Sheedy, P.F., \& Jensen, M.D. (2000). Measuring leg muscle and fat mass in humans: Comparison of CT and dualenergy X-ray absorptiometry. Journal of Applied Physiology, 88, 452-456.

Lohman T.G. (1992). Advances in body composition assessment. Champaign, IL: Human Kinetics.

Lohman, T.G. (1996). Dual energy X-ray absorptiometry. In: A.F. Roche, S.B. Heymsfield, \& T.G. Lohman (Eds.). Human Body Composition, 63-78. Champaign, IL: Human Kinetics.

Lohman, T.G., \& Chen, Z. (2005). Dual-energy X-ray absorptiometry. In: Heymsfield, S.B., Lohman, T.G., Wang, Z., editors. Human body composition: Methods and findings. Champaign, IL: Human Kinetics; 2005. pp. 63-77.

Lohman, T.G., Houtkooper, L., \& Going, S. (1997). Body fat measurement goes hightech: Not all are created equal. ACSM's Health \& Fitness Journal, 1(1), 30-35.

Lohman, M., Tallroth, K., Kettunen, J.A., \& Marttinen, M.T. (2009). Reproducibility of dual-energy X-ray absorptiometry total and regional body composition measurements using different scanning positions and definitions of regions. Metabolism Clinical and Experimental, 58, 1663-1668.

Lukaski, H.C. (1987). Methods for the assessment of human body composition: Traditional and new. American Journal of Clinical Nutrition, 46(4), 537-556.

Lukaski, H.C. (1989). Use of bioelectrical impedance analysis to assess human body composition: A review. In: Livingston GE, ed. Nutritional status assessment of the individual. Trumbull, CT: Food \& Nutrition Press, 189-204.

Mazess, R.B., Barden, H.S., Bisek, J.P., \& Hanson, J. (1990). Dual-energy X-ray absorptiometry for total-body and regional bone-mineral and soft-tissue composition. The American Journal of Clinical Nutrition, 51, 1106-112.

Mitsiopoulos, N., Baumgartner, R.N., Heymsfield, S.B., Lyons, W., Gallagher, D., \& 
Ross, R. (1998). Cadaver validation of skeletal muscle measurement by magnetic resonance imaging and computerized tomography. Journal of Applied Physiology, $85,115-122$.

Modica, M.J., Kanal, K.M., Gunn, M.L. (2011). The obese emergency patient: Imaging challenges and solutions. Radiographics, 31, 811-823.

Moon, J.R., Stout, J.R., Smith-Ryan, A.E., Kendall, K.L., Fukuda, D.H., Cramer, J.T., \& Moon, S.E. (2013). Tracking fat-free mass changes in elderly men and women using single-frequency bioimpedance and dual-energy X-ray absorptiometry: A four-compartment model comparison. European Journal of Clinical Nutrition, 67, S40-S46.

Mourtzakis, M., Prado, C.M.M., Lieffers, J.R., Reiman, T., McCargar, L.J., \& Baracos, V.E. (2008). A practical and precise approach to quantification of body composition in cancer patients using computed tomography images acquired during routine care. Applied Physiology, Nutrition, and Metabolism, 33, $997-$ 1006.

Nana, A., Slater, G.J., Stewart, A.D., \& Burke, L.M. (2014). Methodology review: Using dual-energy X-ray absorptiometry (DXA) for the assessment of body composition in athletes and active people. International Journal of Sport Nutrition and Exercise Metabolism, 24, 198-215.

National Cancer Institute. (2013, July 16). Computed tomography (CT) scans and cancer. http://www.cancer.gov/about-cancer/diagnosis-staging/ct-scans-fact-sheet\#q5.

Nelson, M. E., Fiatarone, M. A., Layne, J. E., Trice, I., Economos, C. D., Fielding, R. A., Ma, R., Pierson, R.N., \& Evans, W. J. (1996). Analysis of body-composition techniques and models for detecting change in soft tissue with strength training. The American Journal of Clinical Nutrition, 63(5), 678-686.

Pfeiffer, J.J., Galvao, D.A., Gibbs, Z., Smith, K., Turner, D., Foster, J., Martins, R., \& Newton, R.U. (2010). Strength and functional characteristics of men and women 65 years and older. Rejuvination Research, 13(1), 75-82.

Pietrobelli, A., Formica, C., Wang, Z., \& Heymsfield, S.B. (1996). Dual-energy X-ray absorptiometry body composition model: Review of physical concepts. American Journal of Physiology, 271, E941-E951.

Pietrobelli, A., Faith, M.S., Allison, D.B., Gallagher, D., Chiumello, G., \& Heymsfield, S.B. (1998). Body mass index as a measure of adiposity among children and adolescents: A validation study. The Journal of Pediatrics, 132(2), 204-210.

Pineau, J.C., Filliard, J.R., \& Bocquet, M. (2009). Ultrasound techniques applied to body fat measurement in male and female athletes. Journal of Athletic Training, 44(2), 142-147.

Prevrhal, S., Lu, Y., Genant, H. K., Toschke, J. O., \& Shepherd, J. A. (2005). Towards standardization of dual X-ray absorptiometry (DXA) at the forearm. Calcified Tissue International, 76(5), 348-354.

Prior, B. C., KJ; Modlesky, CM; Evans, EM; Sloniger, MA; Saunders, M; Lewis, RD. (1997). In vivo validation of whole body composition estimates from dual-energy X-ray absorptiometry. Journal of Applied Physiology, 83(2), 623-630. 
Roche, A.F., Hemsfield, S.B., Lohman, T.G. (1996). Human body composition. Champaign, IL: Human Kinetics Publishers.

Rothney, M.P., Martin, F.P., Xia, Y., Beaumont, M., Davis, C., Ergun, D., Fay, L., Ginty, F., Kochhar, S., Wacker, W., \& Rezzi, S. (2012). Precision of GE Lunar iDXA for the measurement of total and regional body composition in nonobese adults.Journal of Clinical Densitometry: Assessment of Skeletal Health, 15(4), 399-404.

Roubenoff, R., Kehayias, J.J. (1991). The meaning and measurement of lean body mass. Nutrition Reviews, 49, 163-175.

Santos, D. A., Silva, A. M., Matias, C. N., Fields, D. A., Heymsfield, S. B., \& Sardinha, L. B. (2010). Accuracy of DXA in estimating body composition changes in elite athletes using a four compartment model as the reference method. Nutrition \& Metabolism, 7(22), 7075-7077.

Segal, K.R., Van Loan, M., Fitzgerald, P.I., Hodgdon, J.A., \& Van Itallie, T.B. (1988). Lean body mass estimation by bioelectrical impedance analysis: A four-site crossvalidation study. American Journal of Clinical Nutrition, 47, 7-14.

Shen, W., \& Chen, J. (2008). Application of imaging and other noninvasive techniques in determining adipose tissue mass. In: Methods in Molecular Biology, 456, pp. 3954.

Shih, R., Wang, Z., Heo, M., Wang, W., \& Heymsfield, S. B. (2000). Lower limb skeletal muscle mass: development of dual-energy X-ray absorptiometry prediction model. Journal of Applied Physiology, 89(4), 1380-1386.

Sjorstrom, L. (1991). A computer-tomography based multicompartment body composition technique and anthropometric predictions of lean body mass, total and subcutaneous adipose tissue. International Journal of Obesity, 15, 19-30.

Sjorstrom, L., Kvist, H., Cederblad, A., \& Tylen U. (1986). Determination of total adipose tissue and body fat in women by computed tomography, ${ }^{40} \mathrm{~K}$, and tritium. American Journal of Physiology: Endocrinology and Metabolism, 250, E736E745.

Stanforth, P.R., Crim, B.N., Stanforth, D., \& Stults-Kolehmainen, M.A. (2014). Body composition changes among female NCAA division 1 athletes across the competitive season and over a multiyear time frame. Journal of Strength and Conditioning Research, 28(2), 300-307.

Stewart, A. D. (2001). Assessing body composition in athletes. Nutrition, 17(7), 694-695.

Sutton, L., Scott, M., Wallace, J., \& Reilly, T. (2009). Body composition of English Premier League soccer players: influence of playing position, international status, and ethnicity. Journal of Sports Sciences, 27, 1019-1026.

Toombs, R.J., Ducher, G., Shepherd, J.A., \& De Souza, M.J. (2012). The impact of recent technological advances on the trueness and precision of DXA to assess body composition. Obesity, 20, 30-39.

Visser, M., Fuerst, T., Lang, T., Salamone, L., \& Harris, T.B. (1999). Validity of fanbeam dual-energy X-ray absorptiometry for measuring fat-free mass and leg muscle mass. Journal of Applied Physiology, 87(4), 1513-1520. 
Wang, W., Wang, Z., Faith, M.S., Kotler, D., Shih, R., \& Heymsfield, S.B. (1999). Regional skeletal muscle measurement: Evaluation of new dual-energy X-ray absorptiometry model. Journal of Applied Physiology, 87, 1163-1171.

Wang, Z., Shen, W., Withers, R.T., \& Heymsfield, S.B. (2005). Multicomponent molecular-level models of body composition analysis. In: Heymsfield, S.B., Lohman, T.G., Wang, Z., \& Going, S.B. (2 ${ }^{\text {nd }}$ Ed.) Human Body Composition. Champaign, IL: Human Kinetics.

Wagner, D.R., \& Heyward, V.H. (1999). Techniques of body composition assessment: A review of laboraroty and field methods. Research Quarterly for Exercise and Sport, 70(2), 135-149.

Wells, J.C., \& Fewtrell, M.S. (2006). Measuring body composition. Archives of Disease in Childhood, 91(7), 612-617.

Wittich, A., Oliveri, M.B., Rotemberg, E., \& Mautalen, C. (2001). Body composition of professional football (soccer) players determined by dual X-ray absorptiometry. Journal of Clinical Densitometry, 4, 51-55.

Yim, J.E., Heshka, S., Albu, J., Heymsfield, S., Kuznia, P., Harris, T., \& Gallagher, D. (2007). Intermuscular adipose tissue rivals visceral adipose tissue in independent associations with cardiovascular risk. International Journal of Obesity, 31(9), 1400-1405. 